\title{
Experimental Agents for the Treatment of Atherosclerosis: New Directions
}

This article was published in the following Dove Press journal:

Journal of Experimental Pharmacology

\author{
Errika Voutyritsa' \\ Georgios Kyriakos ${ }^{2}$ \\ Alexandros Patsouras ${ }^{3}$ \\ Christos Damaskos ${ }^{1,4}$ \\ Anna Garmpi ${ }^{5}$ \\ Evangelos Diamantis ${ }^{6}$ \\ Nikolaos Garmpis ${ }^{1,7}$ \\ Spyridon Savvanis $\mathbb{1 D}^{8}$ \\ 'N.S. Christeas Laboratory of \\ Experimental Surgery and Surgical \\ Research, Medical School, National and \\ Kapodistrian University of Athens, \\ Athens, Greece; ${ }^{2}$ Sección de \\ Endocrinología y Nutrición, Hospital \\ General Universitario Santa Lucia, \\ Cartagena, Spain; ${ }^{3}$ Second Department of \\ Internal Medicine, Tzanio General \\ Hospital, Piraeus, Greece; ${ }^{4}$ Renal \\ Transplantation Unit, Laiko General \\ Hospital, Athens, Greece; ${ }^{5}$ First \\ Department of Propedeutic Internal \\ Medicine, Laiko General Hospital, \\ Medical School, National and \\ Kapodistrian University of Athens, \\ Athens, Greece; ${ }^{6}$ Department of \\ Endocrinology and Diabetes Center, \\ G. Gennimatas General Hospital, Athens, \\ Greece; ${ }^{7}$ Second Department of \\ Propedeutic Surgery, Laiko General \\ Hospital, Medical School, National and \\ Kapodistrian University of Athens, \\ Athens, Greece; ${ }^{8}$ Internal Medicine \\ Department, Elpis General Hospital, \\ Athens, Greece
}

Correspondence: Evangelos Diamantis $\mathrm{Tel}+302132032000$

$\mathrm{Fax}+302107705980$

Email Vaggelisd0I@gmail.com

\begin{abstract}
Cardiovascular and related metabolic disorders constitute a worldwide health challenge. Atherosclerosis is a chronic inflammatory condition based on both dyslipidemia and inflammation. Therefore, even when dyslipidemia is controlled, the risk of atherosclerosis remains. Among the most efficient inflammatory mediators used as therapeutic tools in cardiovascular disease are the interleukins, which are pro-inflammatory mediators like cytokines. Moreover, a protein kinase inhibitors, p38 mitogen-activated protein kinase (MAPK) inhibitor, and an inhibitor of a leukocyte adhesion molecule, P-Selectin, have also presented therapeutic potential for this disorder. Colchicine, being an inexpensive therapeutic option, has been proved to be suitable for the prevention of atherosclerosis. In this review, we summarize all the studies, from 2010 to 2020, in which treatment approaches based on the agents mentioned above are evaluated in the management of atherosclerosis.
\end{abstract}

Keywords: cytokines, interleukins, losmapimod, colchicine, p-selectin, inclacumab

\section{Introduction}

Atherosclerosis occurs when macrophage and elements of fat, mostly cholesterol and its derivatives, are formed in the artery walls, limiting blood flow. ${ }^{1,2}$ Atherosclerosis is a disease, which develops gradually. Mild atherosclerosis usually does not have any symptoms. However, the progressed atherosclerosis leads to formation of blood clot, which completely blocks blood flow and results in a heart attack, stroke or coronary artery disease (CAD). ${ }^{1}$

Atherosclerosis is a progressive disease and is associated with several risk factors such as hypercholesterolemia, high blood pressure, diabetes, obesity, high triglycerides, genetic abnormalities, as well as life style factors including stress, physical inactivity and tobacco smoking. All the factors presented may cause damage to the inner layer of an artery, leading to atherosclerosis. ${ }^{3,4}$

It is common knowledge that hypercholesterolemia is the most important pathogenic factor in both the initiation and progression of atherosclerosis. ${ }^{5}$ Therefore, several immunotherapeutic approaches and strategies, interfering with lipoprotein metabolism and vascular cell function, have been developed as potential therapeutic tools. ${ }^{6}$

Specifically, cytokines constitute a group of proteins that modulate immunity. Certain cytokines have been proved to modulate the propagation of atherosclerosis. Interleukins, for example, a type of cytokines, mediate signaling between white blood cells. Therefore, numerous cytokines have been identified as potential therapeutic targets. $^{7-9}$ Anti-CD3 antibodies and approaches targeting T-cells, are also

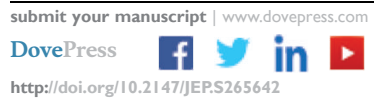


identified as potent immunosuppressive factors, as they modulate the CD3/T-cell receptor complex. ${ }^{10,11}$ Additionally, co-stimulatory signals of T-cell activation constitute suitable targets for immunological interventions. $^{12-14}$ Various antigens have also been observed to initiate immune responses which modulate atherosclerosis, such as bacterial and viral antigens, as well as self-antigens that have been altered. Immune responses to the majority of these antigens modulate disease propagation. ${ }^{15,16}$

This review will focus on the application of a range of experimental agents, which modulate immune responses relevant to arteriosclerosis at several levels. The effectiveness and the safety of these therapeutic tools to address atherosclerosis will be discussed. This review includes clinical trials that have studied the development and use of these agents in preclinical studies and in patients for the time period from 2010 to 2020 .

\section{Materials and Methods}

\section{Selection}

A systematic research was conducted in databases including PubMed, EMBASE and Cochrane for a time period from 2010 to 2020. During this research, keywords such as atherosclerosis, lipid lowering agents, cytokines, interleukins, protein kinase inhibition, Losmapimod, Colchicine, p-Selectin, Inclacumab and NLRP3 inhibitors were used. The PRISMA approach was applied for the selection of the published articles included in the review. The group of interest included subjects presenting atherosclerosis at various levels. The reduction of atherosclerotic plaque and the limitation of their progression were demonstrated as the successful endpoints of these studies. For articles to be selected, the suggestion of cytokines, interleukins, protein kinase inhibitors, Losmapimod, Colchicine, p-Selectin, Inclacumaband NLRP3 inhibitors should be the treatment strategies. Furthermore, studies also included several information about the sample of patients presenting atherosclerosis such as age or gender, as well as information concerning diagnostic criteria, and the baseline medical treatment. Review articles and case reports were not considered eligible for the purpose of this review. Moreover, articles presenting only abstracts or not being completely relevant to the topic were excluded from this review. Duplicates or publications that were not in English language were also excluded. Reference lists of all accessed full-text articles were further searched for sources of potentially relevant information. A total of 78 records were identified. Following removal of the non-suitable articles, 22 records remained. The full-text articles assessed for eligibility were 22 and none of them was excluded.

\section{Results \\ Cytokines}

Research on atherosclerosis is based on identifying all the risk factors for atherosclerosis, such as increased levels of plasma LDL-cholesterol and decreased levels of plasma HDL-cholesterol. Moreover, risk factors for atherosclerosis constitute several pro-inflammatory mediators, like cytokines. Cytokines are produced in atherosclerotic lesions, mainly by macrophages and their inhibition contributes to atherosclerosis.

In 2011, Ridker et al, started the investigation concerning the ability of interleukin-1 $\beta$ (IL-1 $\beta$ ) inhibition to reduce the rate of cardiovascular conditions in with coronary artery disease. ${ }^{17}$ Interleukins are crucial mediators involved in the systemic inflammatory response. Particularly interleukin-1 (IL-1) is a pro-inflammatory cytokine with a quite important role in the atherothrombotic process. Moreover, Canakinumab is used as a therapeutic tool for inherited IL-1 $\beta$ driven inflammatory conditions. In this study, 17.200 patients with persistent increase of hsCRP were randomized to either placebo or to Canakinumab. All patients enrolled to this study followed up for 4 years and were evaluated for nonfatal myocardial infarction, nonfatal stroke, cardiovascular death, as well as for other cardiovascular events. Importantly, patients treated with 50,150, and $300 \mathrm{mg}$ of Canakinumab presented an optimal balance between safety, tolerability and efficacy in inflammatory conditions.

The possible effect of inflammation on atherosclerosis was examined in mice. Schuett et al studied the potential of glycoprotein 130 (sgp130) and IgG1-Fc (sgp130Fc), natural interleukin-6 (IL-6) transsignaling inhibitors, to reduce atherosclerosis in hypercholesterolemic mice (Table 1, entry 1). ${ }^{18}$ During this study, hypercholesterolemic mice, received a high-fat, and high-cholesterol diet, were randomized to 3 groups. The study began after 12 weeks of feeding. Mice in 1 of the groups were injected twice weekly with sgp $130 \mathrm{Fc}$, whereas the control group received only the vehicle phosphate-buffered saline (PBS). The results were evaluated by the tissue and fasted plasma collected from mice after 12 weeks and 24 weeks of diet, 


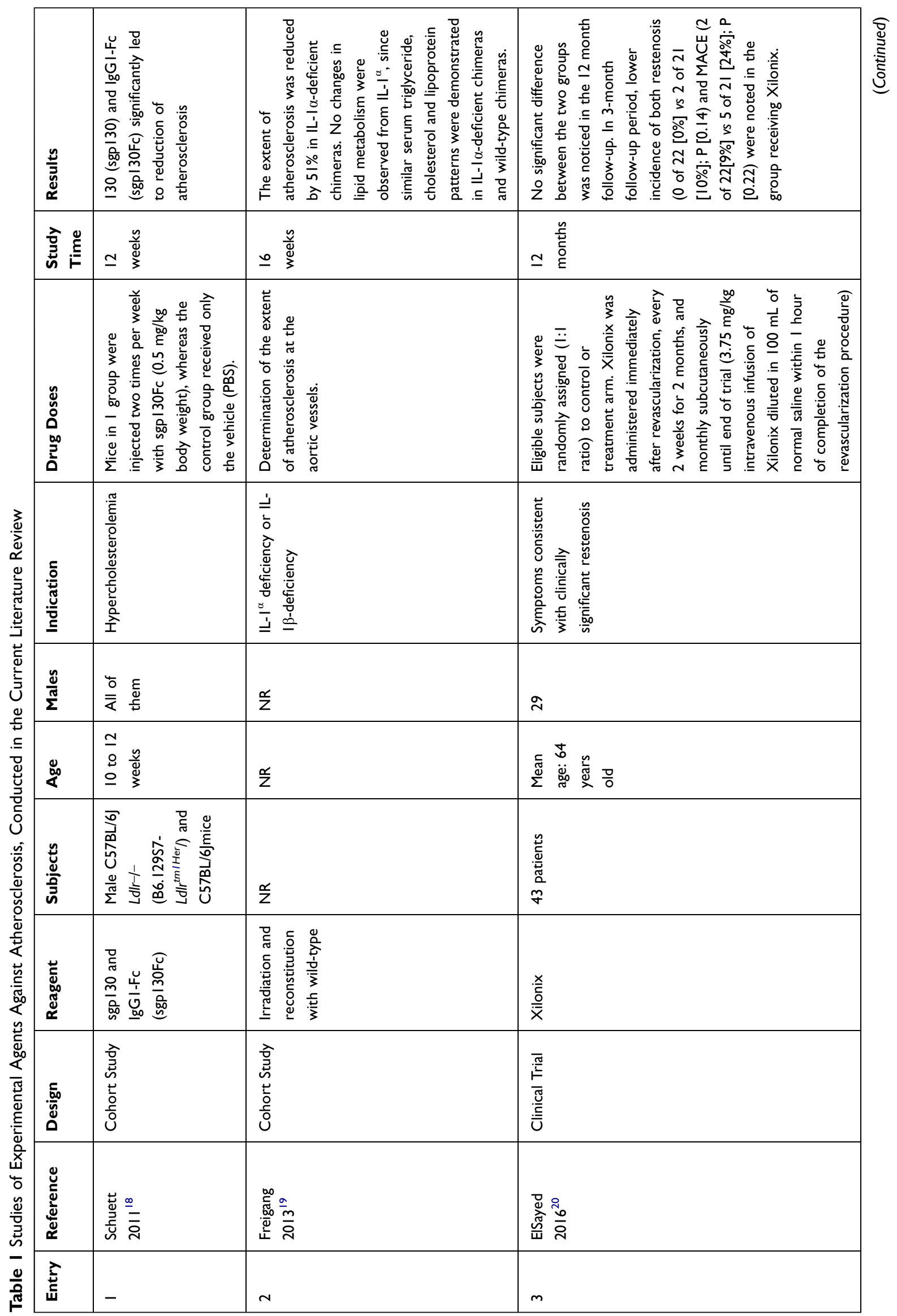




\begin{tabular}{|c|c|c|}
\hline 号 & 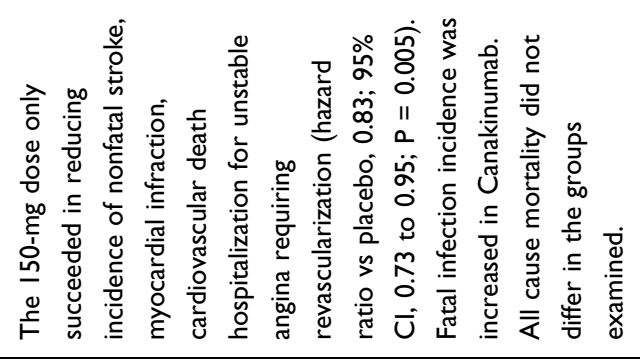 & 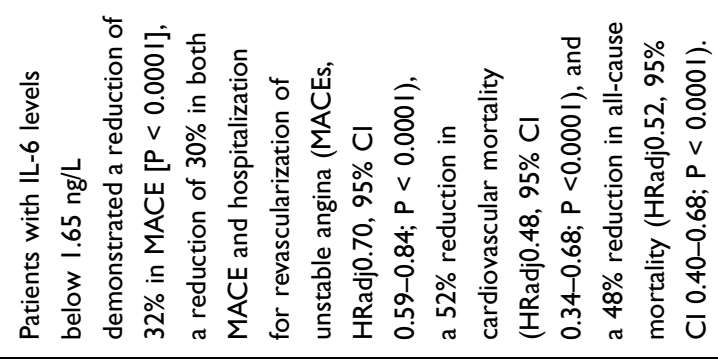 \\
\hline 氞 & 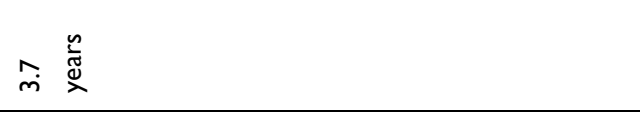 & 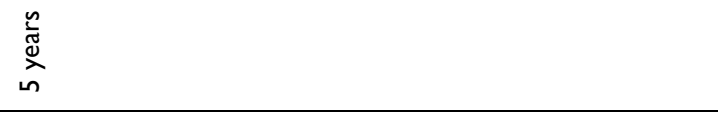 \\
\hline 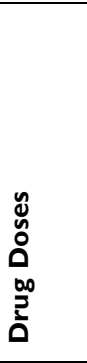 & 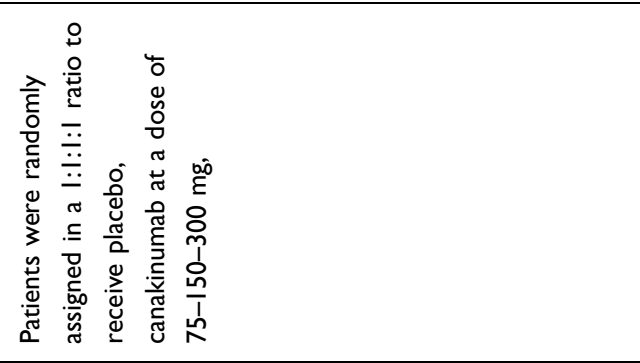 & 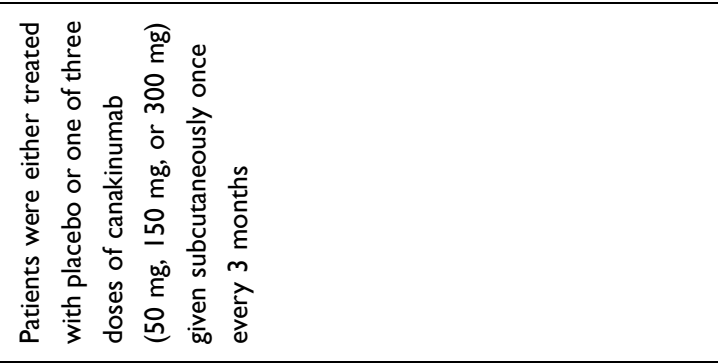 \\
\hline 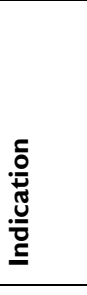 & 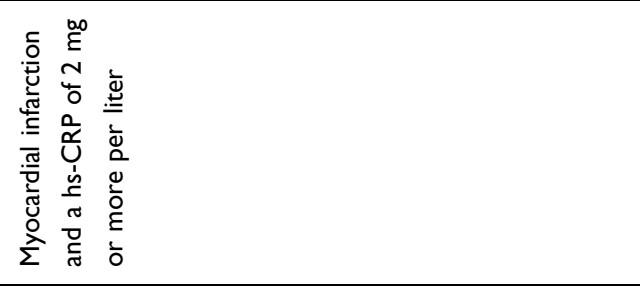 & 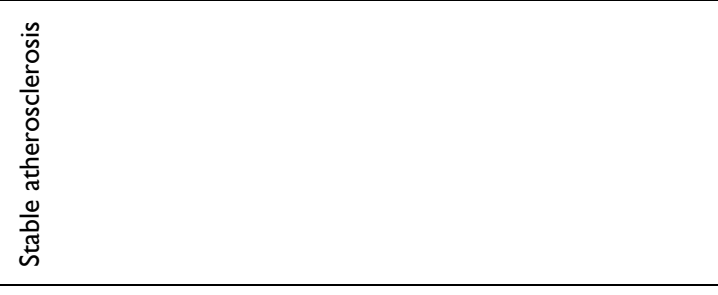 \\
\hline$\frac{\frac{y}{\sigma}}{\tilde{\sigma}}$ & $\underset{\substack{\infty \\
\infty}}{\infty}$ & 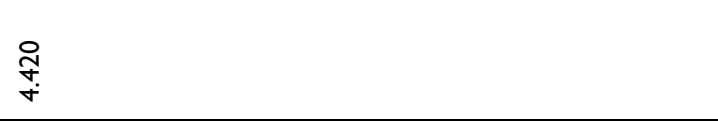 \\
\hline$\underset{\&}{\&}$ & 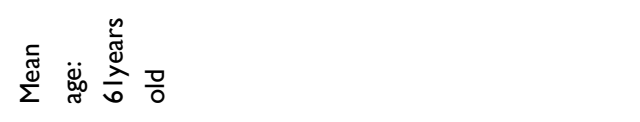 & 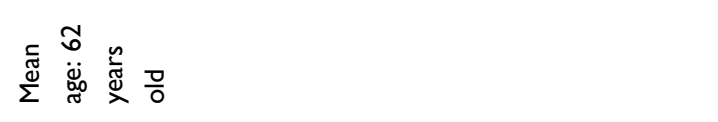 \\
\hline & 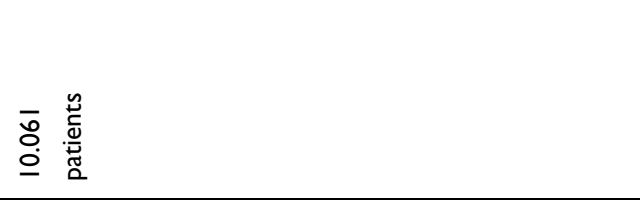 & 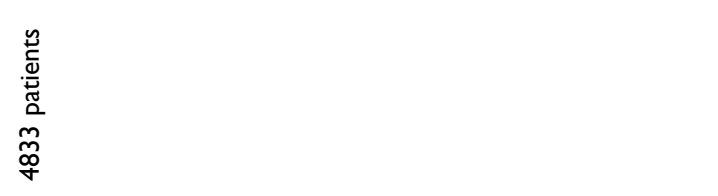 \\
\hline & 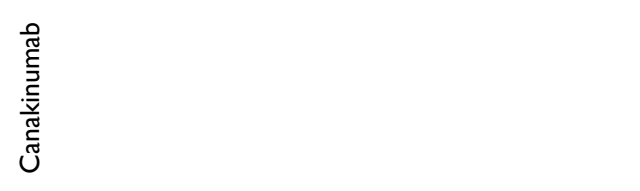 & 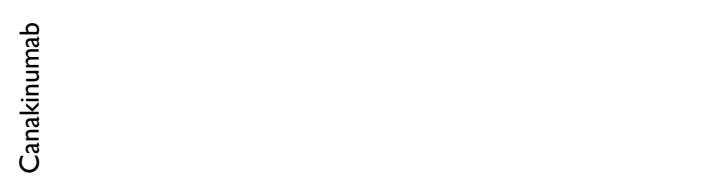 \\
\hline 点 & 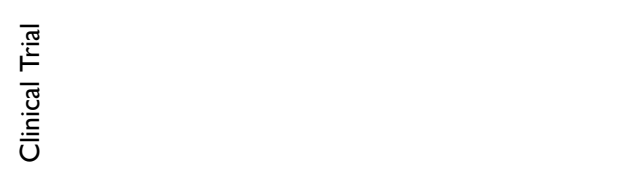 & 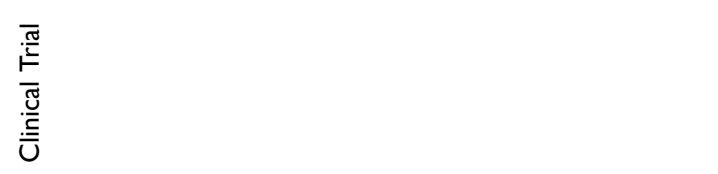 \\
\hline 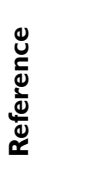 & 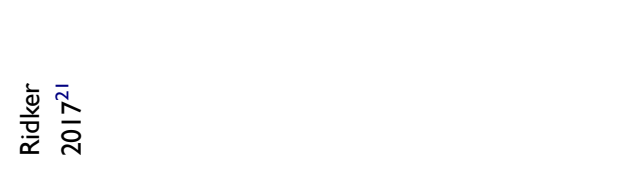 & 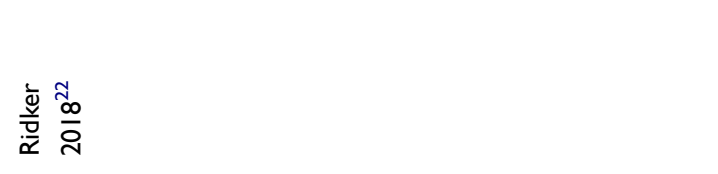 \\
\hline 意 & $\sigma$ & n \\
\hline
\end{tabular}




\begin{tabular}{|c|c|}
\hline 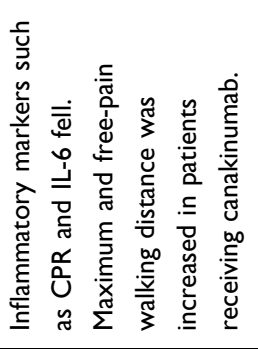 & 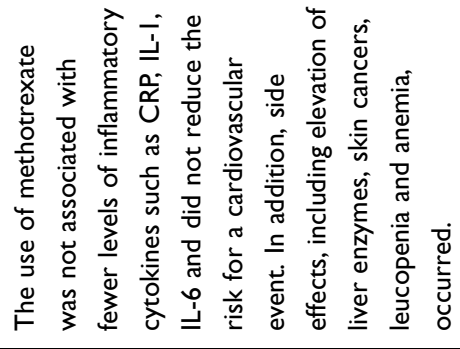 \\
\hline 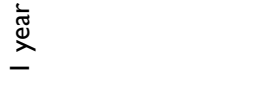 & 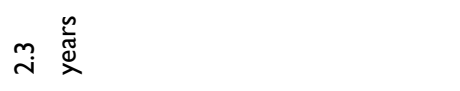 \\
\hline 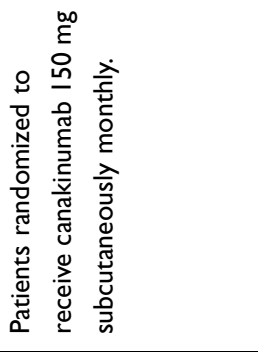 & 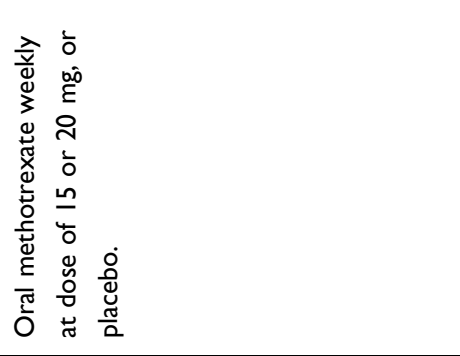 \\
\hline 希 & 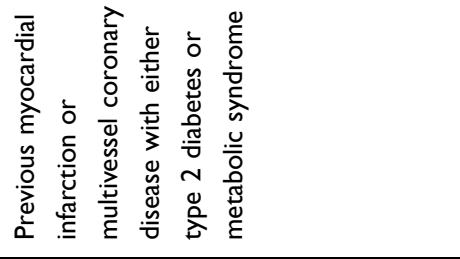 \\
\hline$\stackrel{\circ}{\stackrel{\circ}{\wedge}}$ & $\frac{\circ}{\infty}$ \\
\hline 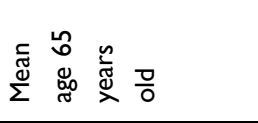 & 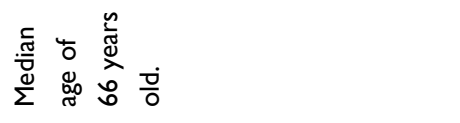 \\
\hline$\stackrel{\infty}{m}$ & 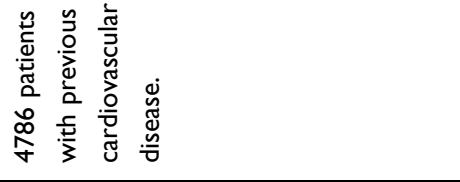 \\
\hline 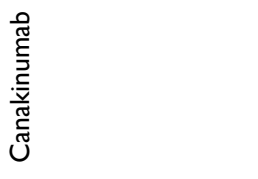 & 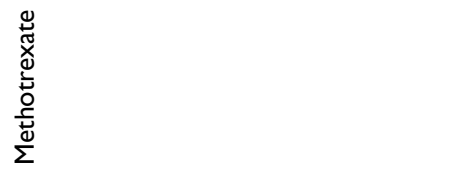 \\
\hline 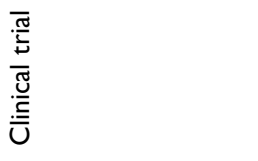 & 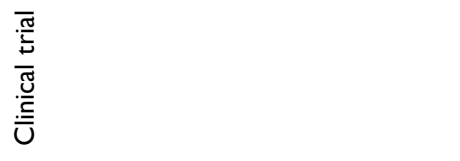 \\
\hline 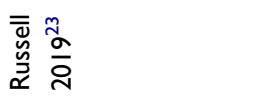 & 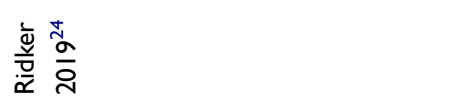 \\
\hline 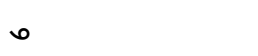 & $\wedge$ \\
\hline
\end{tabular}




\begin{tabular}{|c|c|}
\hline 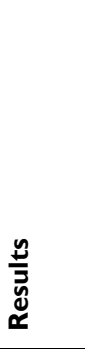 & 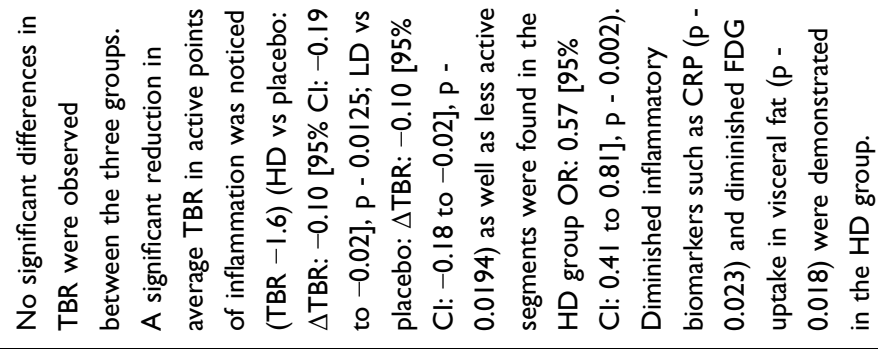 \\
\hline 突 & 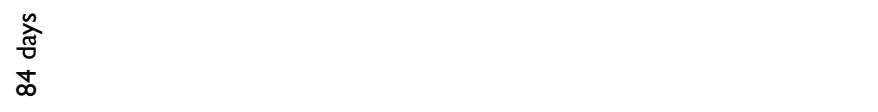 \\
\hline 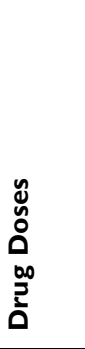 & 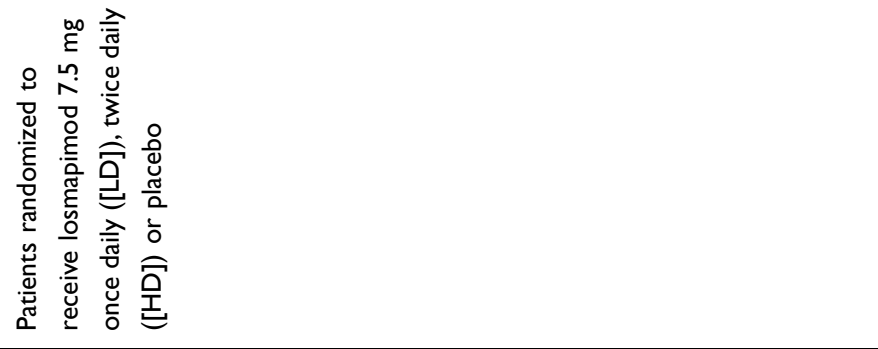 \\
\hline & 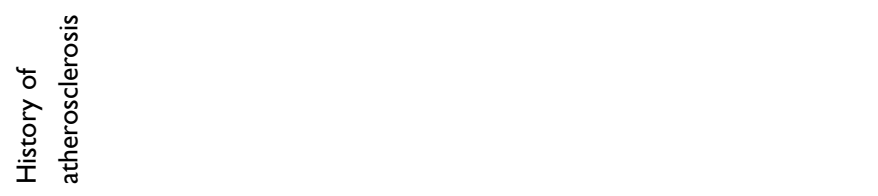 \\
\hline$\frac{\tilde{y}}{\frac{0}{\pi}}$ & $\stackrel{\alpha}{Z}$ \\
\hline 品 & 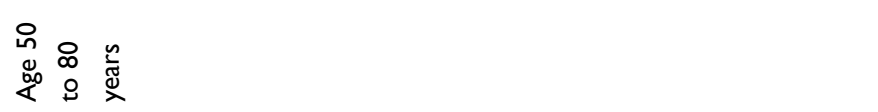 \\
\hline 嵌 & 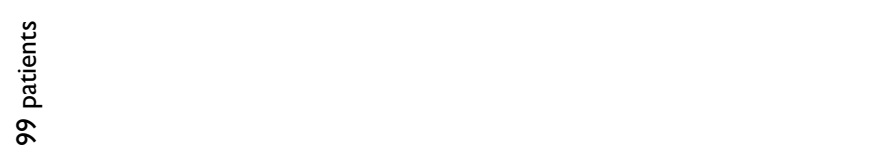 \\
\hline 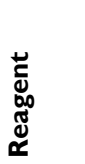 & 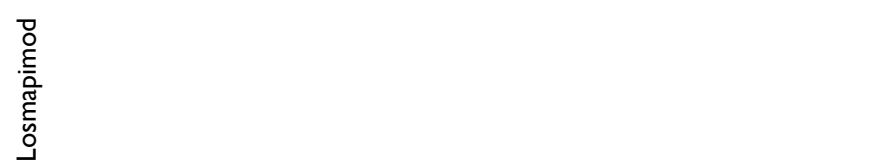 \\
\hline פั. & 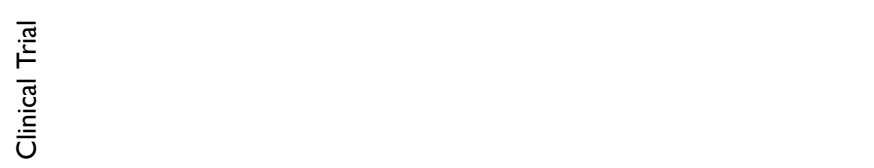 \\
\hline 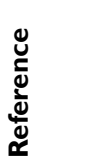 & 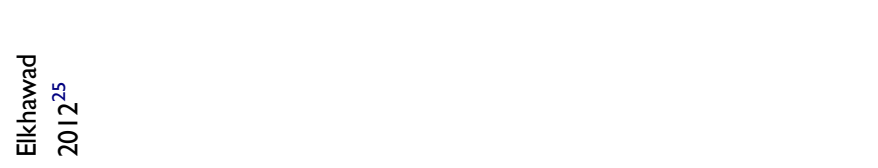 \\
\hline 密 & $\infty$ \\
\hline
\end{tabular}




\begin{tabular}{|c|c|c|}
\hline 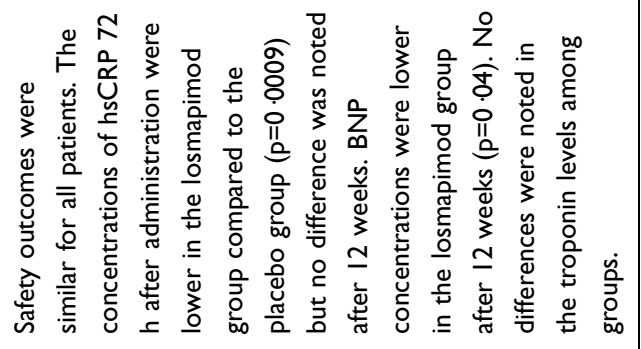 & 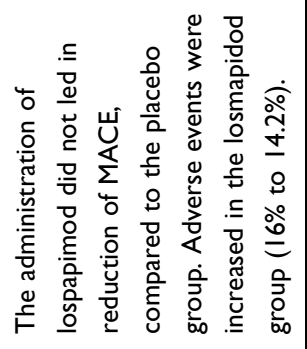 & 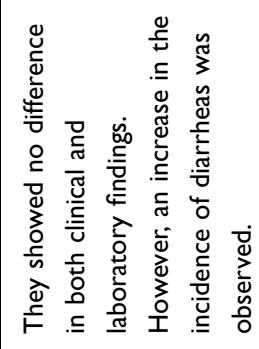 \\
\hline 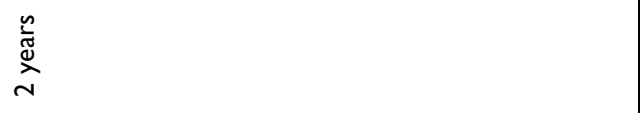 & 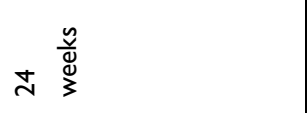 & $\stackrel{o}{Z}$ \\
\hline 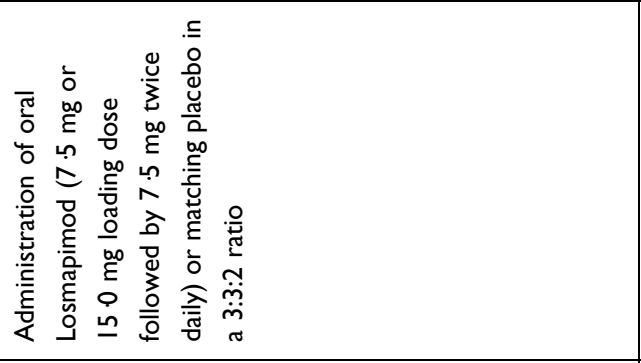 & 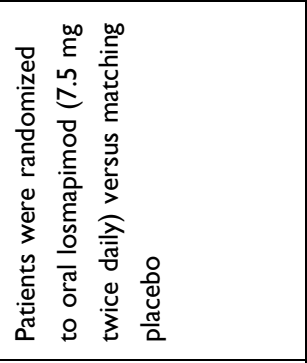 & 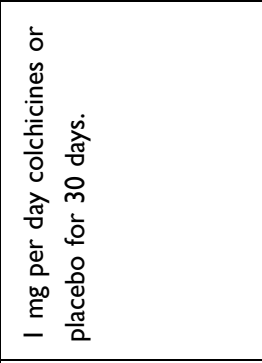 \\
\hline 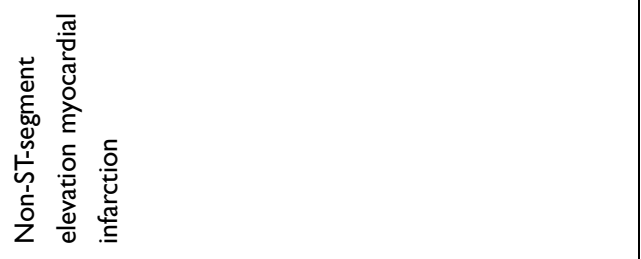 & 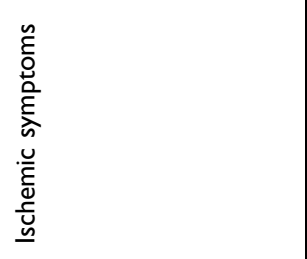 & 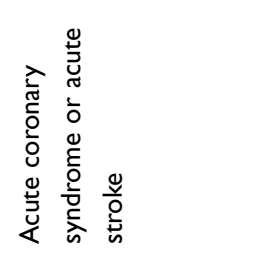 \\
\hline$\stackrel{\sim}{q}$ & $\begin{array}{l}\text { to } \\
\text { oे }\end{array}$ & $\bar{r}$ \\
\hline 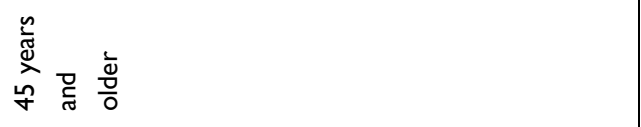 & 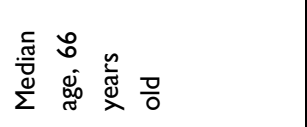 & 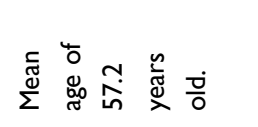 \\
\hline 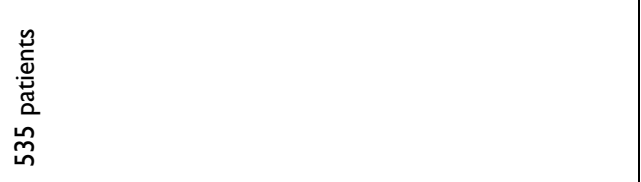 & 莽 & 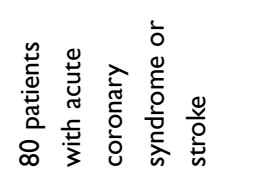 \\
\hline 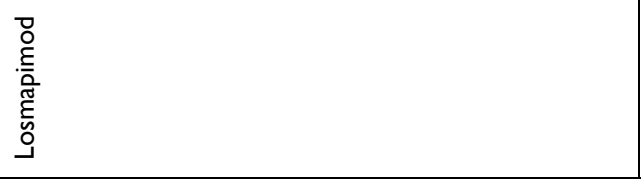 & 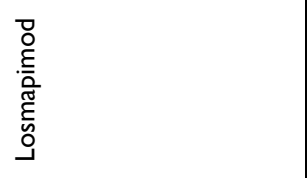 & 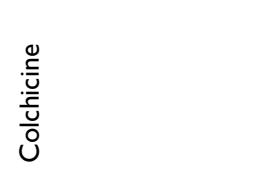 \\
\hline 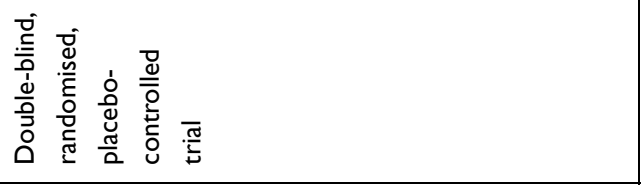 & 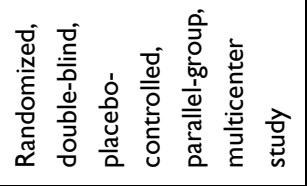 & 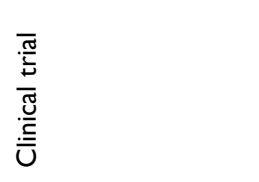 \\
\hline 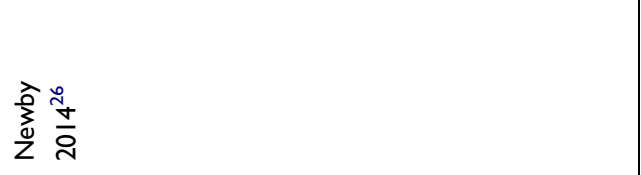 & 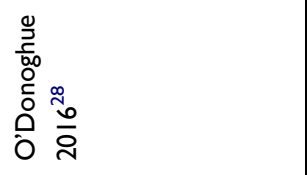 & 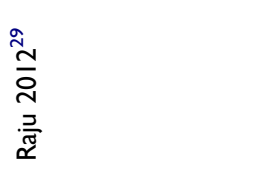 \\
\hline$\alpha$ & 으 & $=$ \\
\hline
\end{tabular}




\begin{tabular}{|c|c|c|c|}
\hline 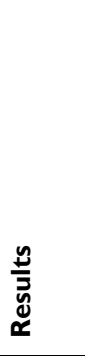 & 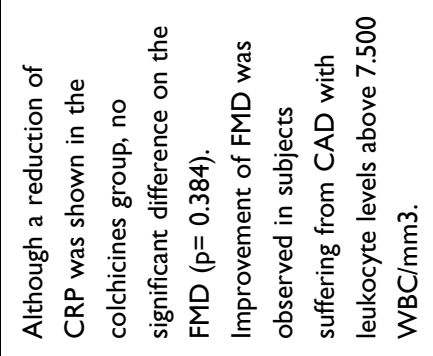 & 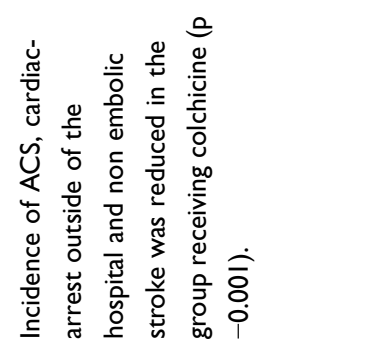 & 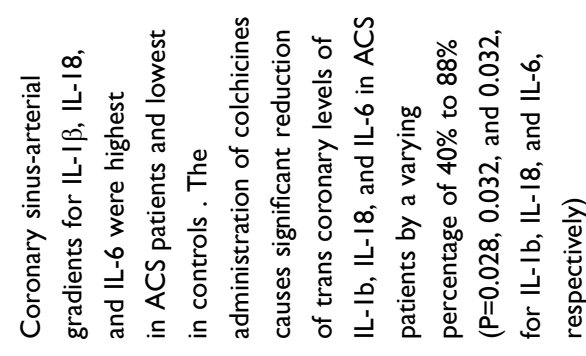 \\
\hline 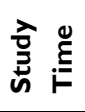 & $\infty \stackrel{\stackrel{n}{0}}{\stackrel{0}{\varepsilon}}$ & $\begin{array}{l}\stackrel{n}{\bar{N}} \\
\text { న } \\
m\end{array}$ & 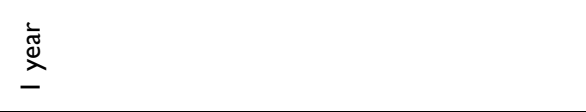 \\
\hline 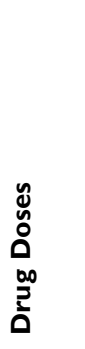 & 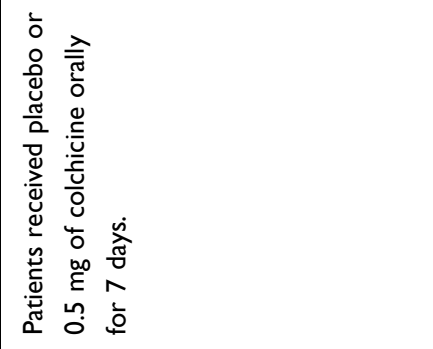 & 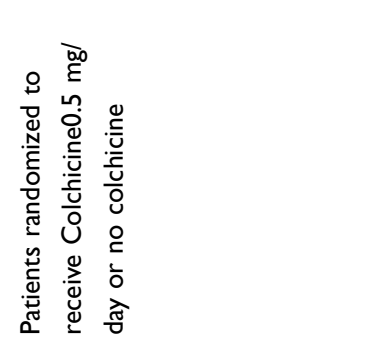 & 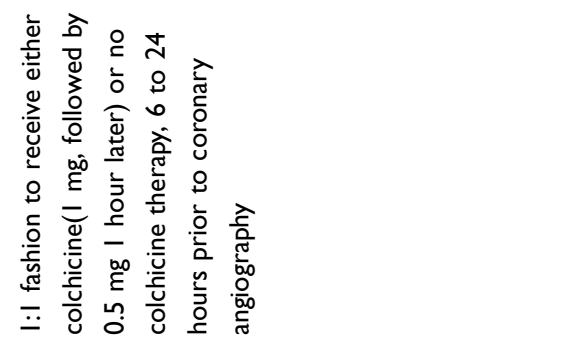 \\
\hline 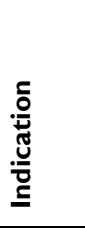 & 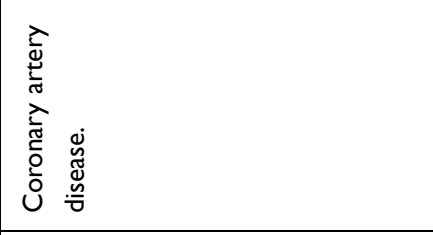 & 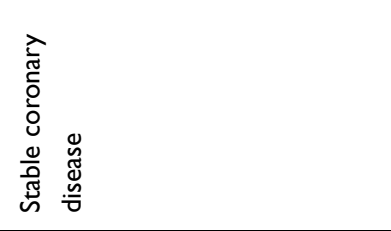 & 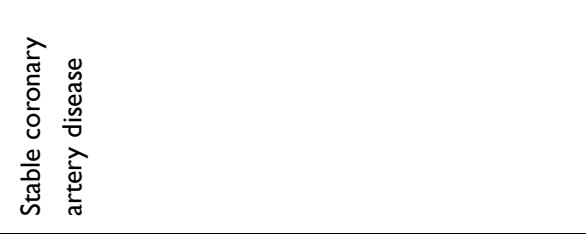 \\
\hline$\frac{\tilde{\omega}}{\Sigma}$ & $\grave{\sim}$ & $\tilde{F}$ & $\stackrel{\star}{\Sigma}$ \\
\hline 总 & 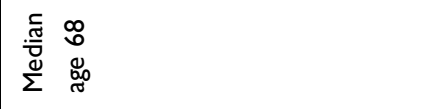 & 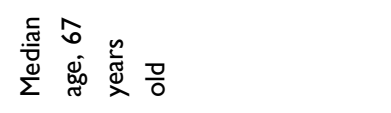 & 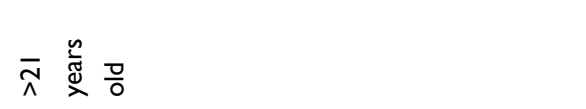 \\
\hline 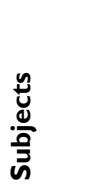 & 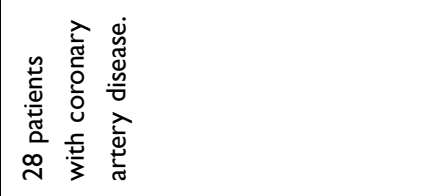 & 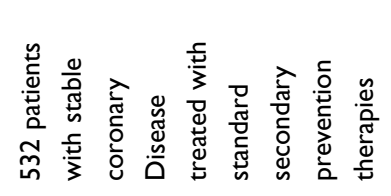 & 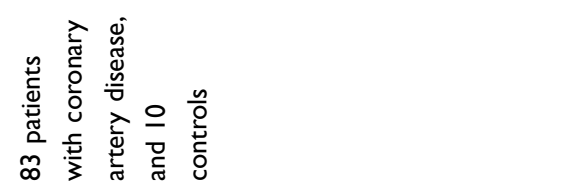 \\
\hline 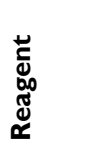 & 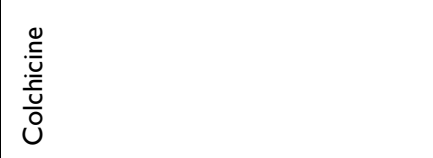 & 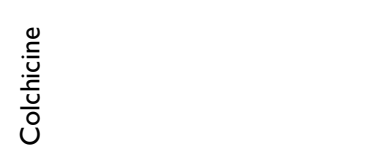 & 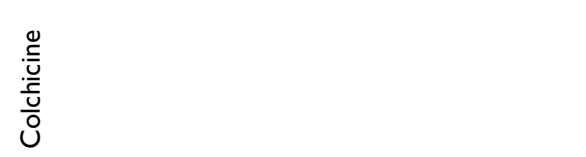 \\
\hline 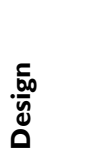 & 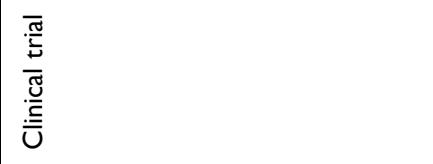 & 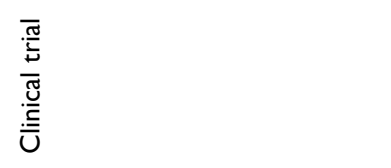 & 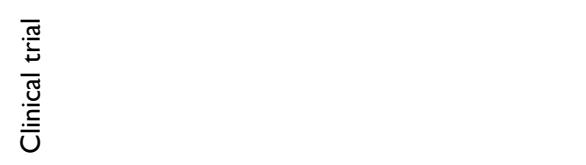 \\
\hline 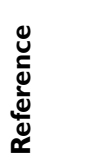 & 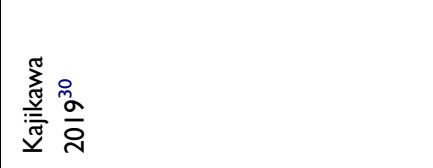 & 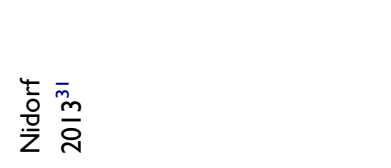 & 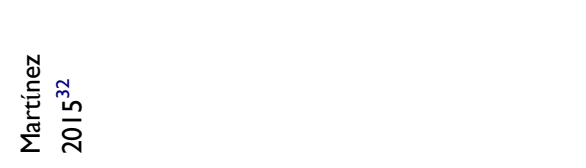 \\
\hline 站 & $\simeq$ & $\underline{m}$ & \pm \\
\hline
\end{tabular}




\begin{tabular}{|c|c|c|}
\hline 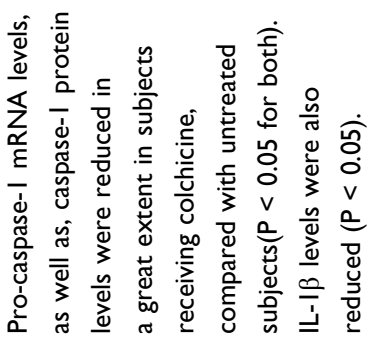 & 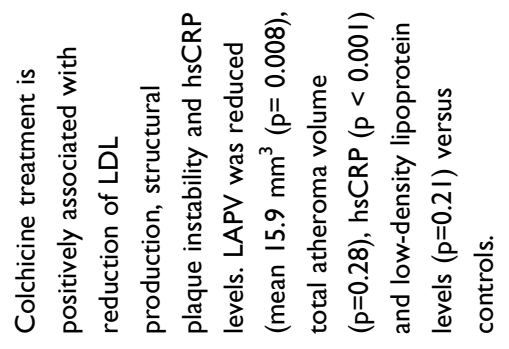 & 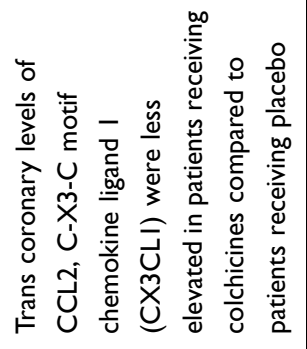 \\
\hline$\dot{z}$ & 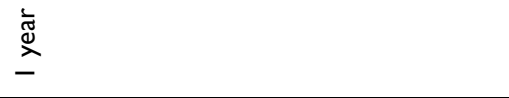 & $\frac{\alpha}{Z}$ \\
\hline 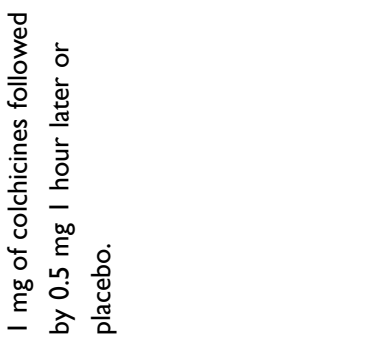 & 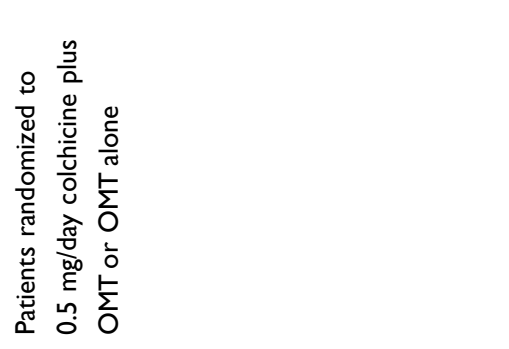 & 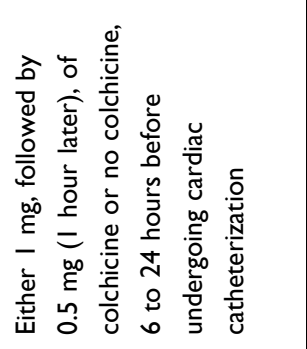 \\
\hline 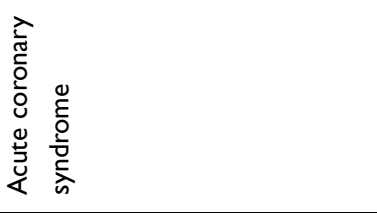 & 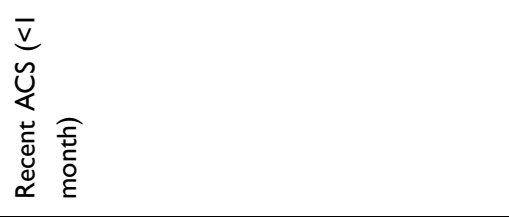 & 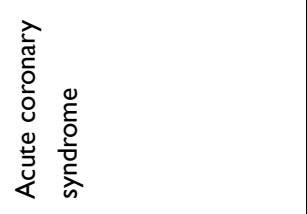 \\
\hline$\underline{n}$ & $\widehat{\sigma}$ & 兴 \\
\hline 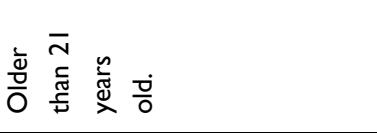 & 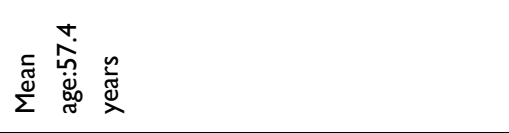 & 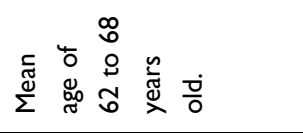 \\
\hline 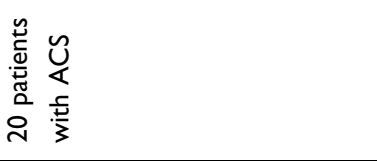 & 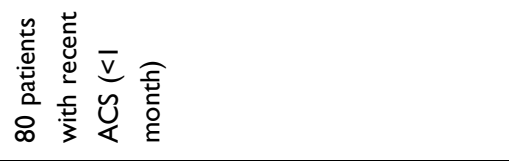 & 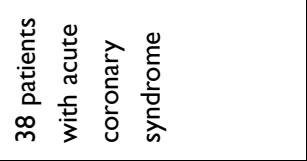 \\
\hline & 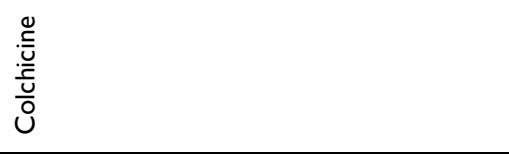 & $\begin{array}{l}\frac{0}{0} \\
\frac{0}{\bar{v}} \\
\frac{0}{0}\end{array}$ \\
\hline 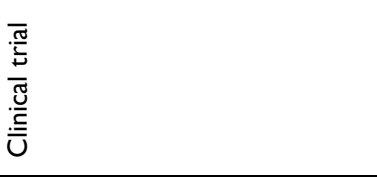 & 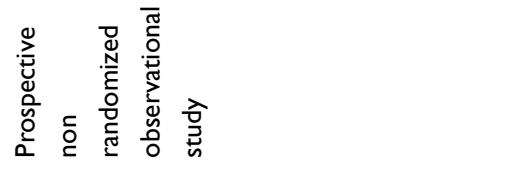 & 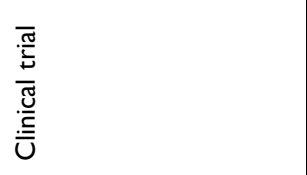 \\
\hline 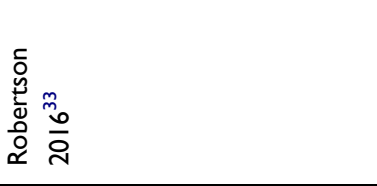 & 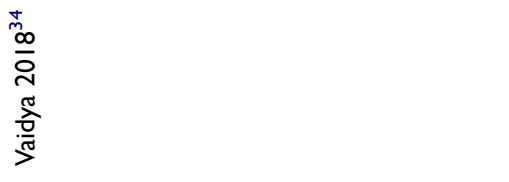 & 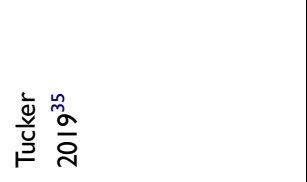 \\
\hline$\underline{n}$ & $\underline{0}$ & $\simeq$ \\
\hline
\end{tabular}




\begin{tabular}{|c|c|c|}
\hline 蒿 & 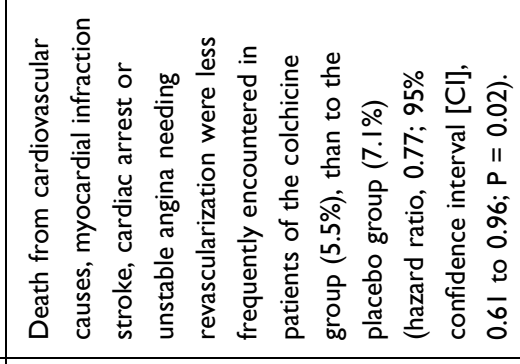 & 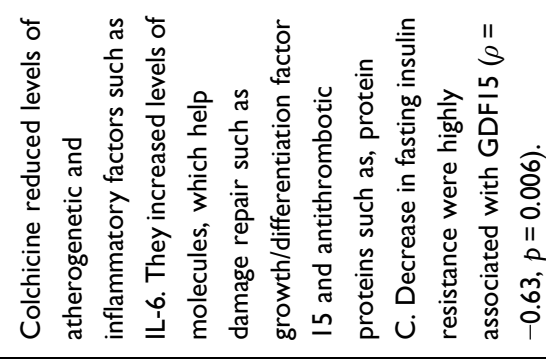 \\
\hline 高 & 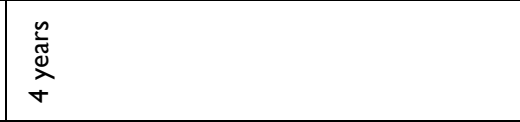 & 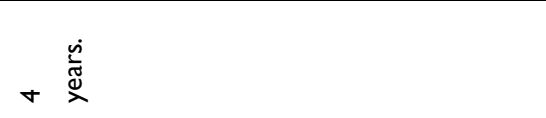 \\
\hline 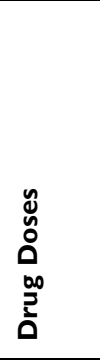 & 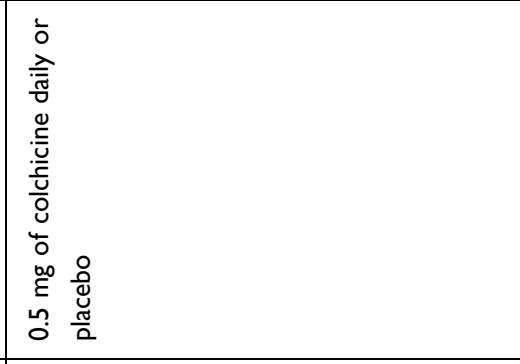 & 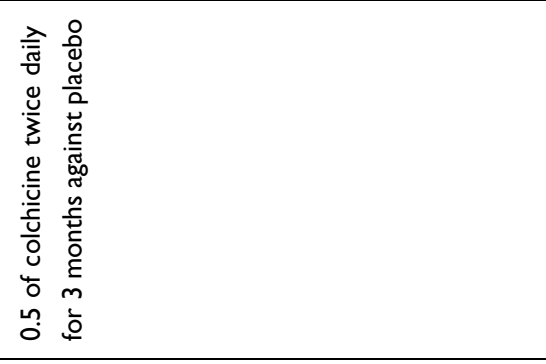 \\
\hline 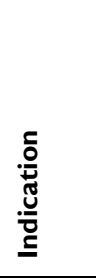 & 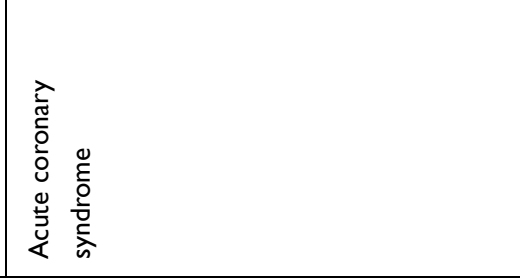 & 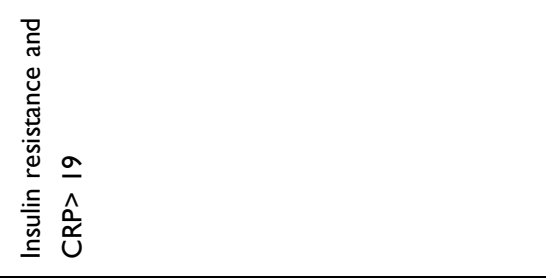 \\
\hline$\frac{\tilde{\omega}}{\frac{\sigma}{\sigma}}$ & 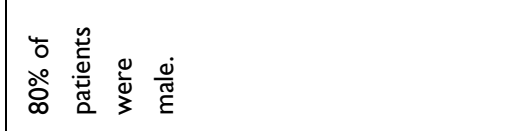 & 总 \\
\hline 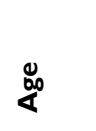 & 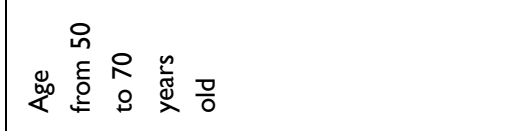 & 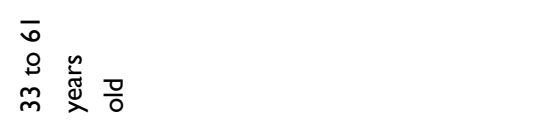 \\
\hline & 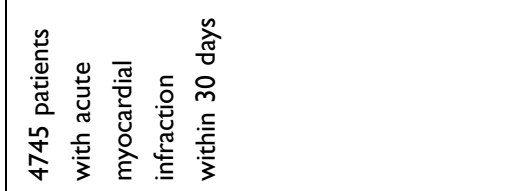 & 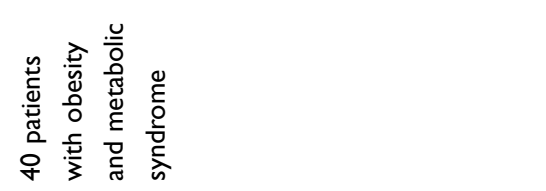 \\
\hline 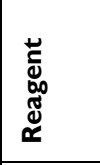 & 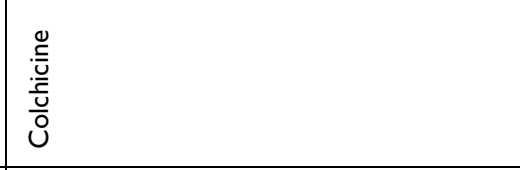 & 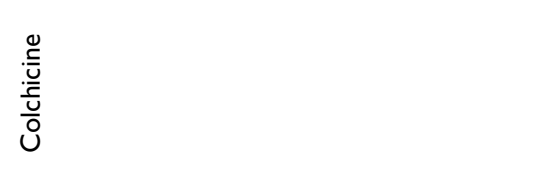 \\
\hline 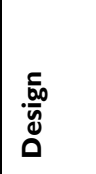 & 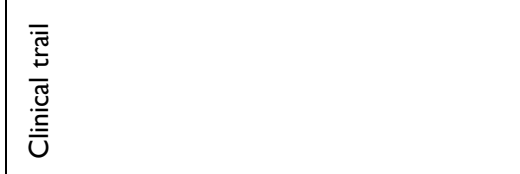 & 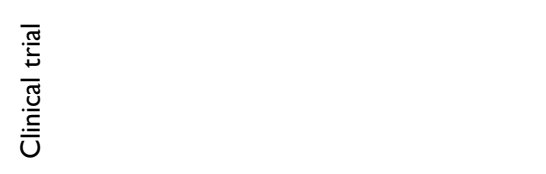 \\
\hline 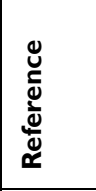 & 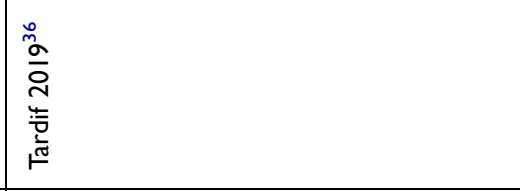 & 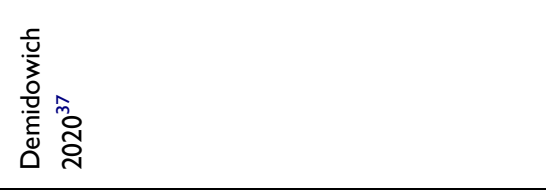 \\
\hline 意 & $\stackrel{\infty}{ }$ & $\underline{a}$ \\
\hline
\end{tabular}




\begin{tabular}{|c|c|}
\hline 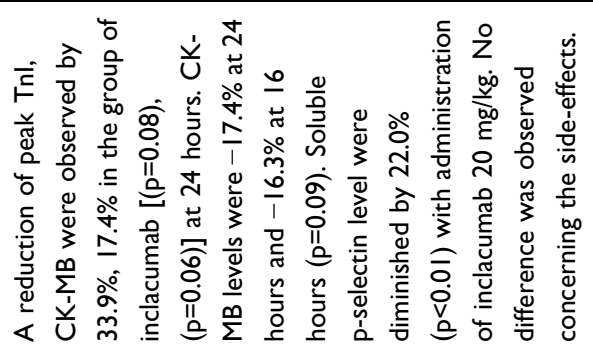 & 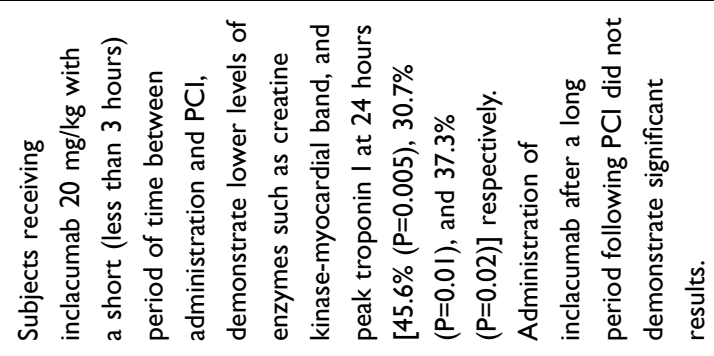 \\
\hline 오 荅 & 오 총 \\
\hline 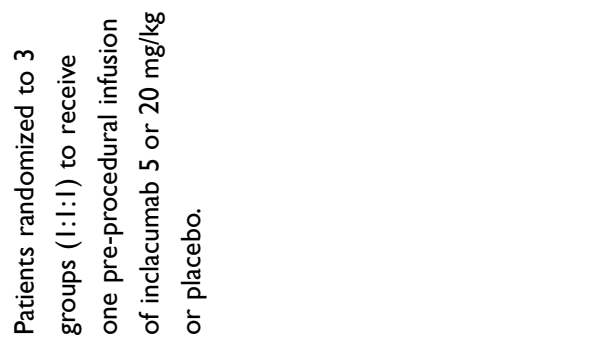 & 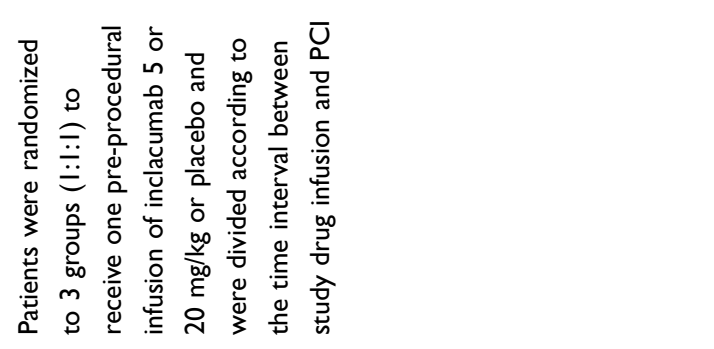 \\
\hline 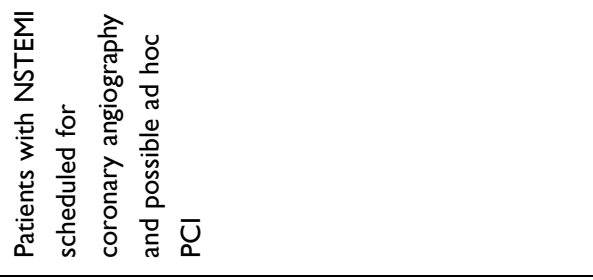 & 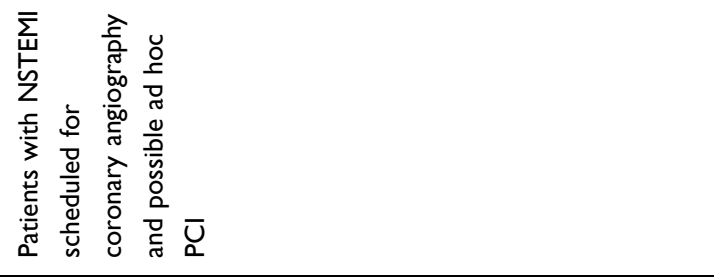 \\
\hline స్ & శ్ \\
\hline 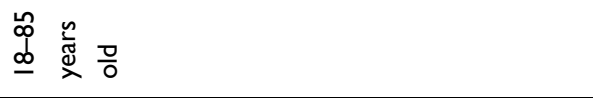 & 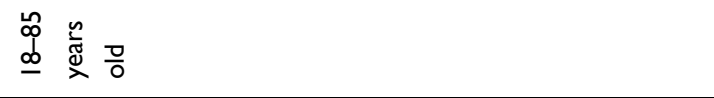 \\
\hline 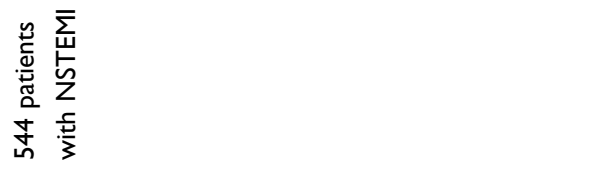 & 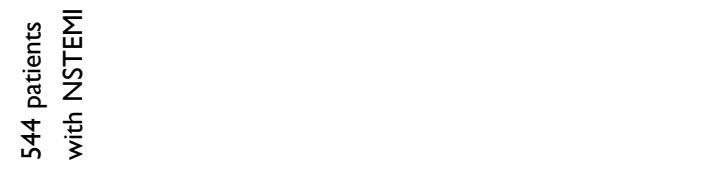 \\
\hline 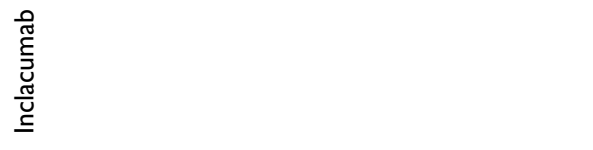 & 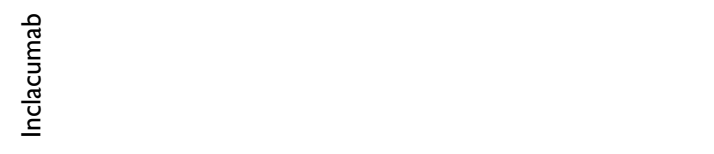 \\
\hline 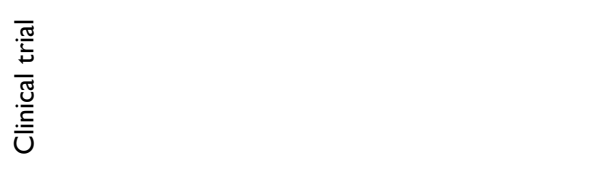 & 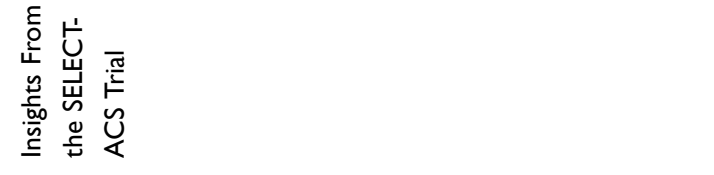 \\
\hline 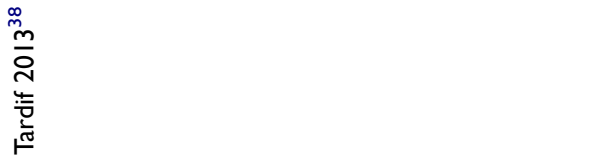 & 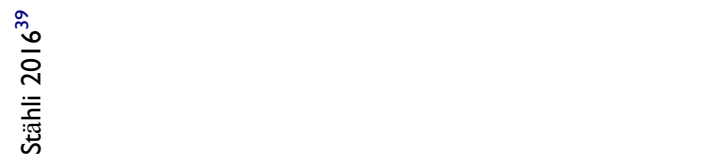 \\
\hline ஜ & $\bar{N}$ \\
\hline
\end{tabular}




\begin{tabular}{|c|c|}
\hline $\begin{array}{l}\stackrel{y}{y} \\
\overline{\vec{y}} \\
\check{y}\end{array}$ & 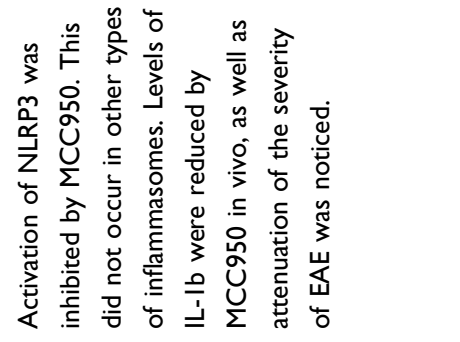 \\
\hline 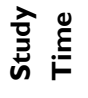 & $\frac{\alpha}{z}$ \\
\hline 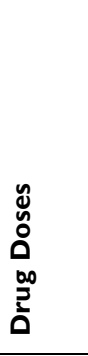 & 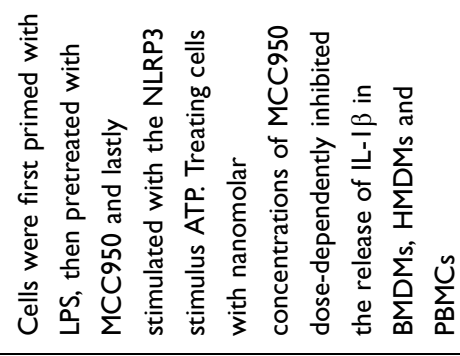 \\
\hline 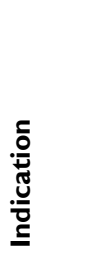 & 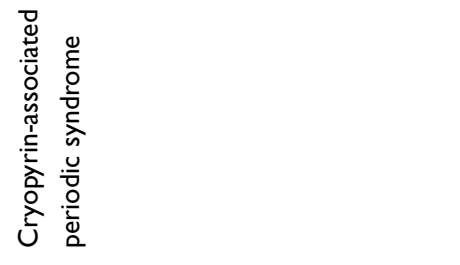 \\
\hline$\frac{\frac{\mathscr{U}}{\pi}}{\Sigma}$ & 号 \\
\hline$\stackrel{8}{8}$ & 号 \\
\hline 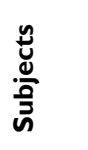 & 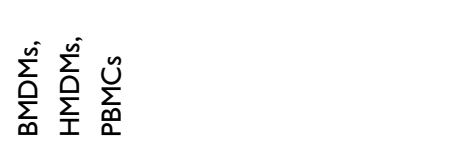 \\
\hline 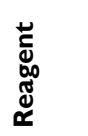 & 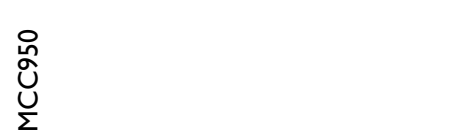 \\
\hline 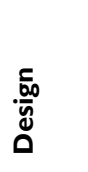 & 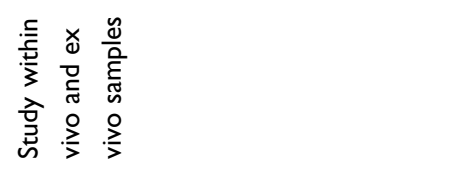 \\
\hline 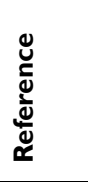 & 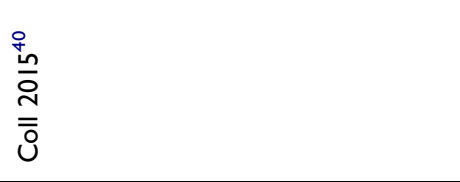 \\
\hline 矛 & $\approx$ \\
\hline
\end{tabular}

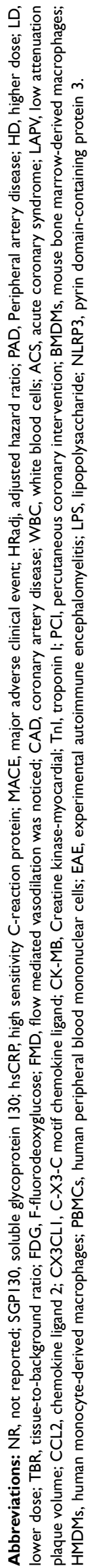

including 12 weeks with sgp130Fc or vehicle injection. According to the results, 130 (sgp130) and IgG1-Fc $(\mathrm{sgp} 130 \mathrm{Fc})$ significantly reduced atherosclerosis in hypercholesterolemic mice without presenting any side effect concerning weight gain and serum lipid levels. Importantly, sgp130Fc treatment resulted in a notable regression of advanced atherosclerosis, indicating that IL6 transsignaling may be therapeutic for atherogenesis in humans. Freigang et al evaluated the correlation between macrophage-derived IL- $1 \alpha$ or IL-1 $\beta$ and atherosclerosis in bone marrow of chimeras (Table 1, entry 2). ${ }^{19}$ In this study, mice deficient in the receptor for low-density lipoprotein were irradiated and reconstituted with IL-1 $\alpha$ deficiency or IL-1 $\beta$-deficiency in bone marrow. Subjects were fed with a high-cholesterol diet for 16 weeks and then the determination of the extent of atherosclerosis at the aortic origin took place. The results showed that atherosclerosis was detected in a significantly reduced level by $51 \%$ in IL- $1 \alpha$-deficient chimeras, compared to IL- $1 \beta$ deficient chimeras, without altering lipid metabolism. These findings indicate that IL- $1 \alpha$ was the predominant inflammatory cytokine, which contributes to atherosclerosis via IL-1R signaling.

In 2016, El Sayed et al evaluated the safety and efficiency of Xilonix, a true human monoclonal antibody targeting IL-1a, when used as a therapeutic agent in patients with vascular disease. They also investigated the efficiency of IL-1a inhibition to reduce superficial femoral artery (SFA) restenosis leading to the reduction of the vascular inflammation-related complications (Table 1, entry 3). ${ }^{20}$ In this trial patients, presenting superficial femoral artery restenosis, were randomized to receive the standard of care arm, or the Xilonix plus the standard of care arm. Xilonix was given to patients immediately after revascularization. After 12 months of treatment, major adverse cardiovascular events as well as target vessel restenosis rates were not significantly different between the groups. However, a lower incidence of restenosis and adverse cardiovascular events in the Xilonix cohort were observed, after 3-month of treatment. Therefore, Xilonix can be considered as a potential safe and effective therapeutic method to cure vessel patency.

Canakinumab was examined in several clinical trials, concerning its effect against inflammatory cytokines. Ridker et al reported their results of their trial, in which the effectiveness of interleukin- $1 \beta$ immunity pathway inhibition in reducing the risk of cardiovascular disease was investigated (Table 1, entry 4). ${ }^{21}$ In this study 10.061 
patients, presenting myocardial infarction and low C-reactive protein level, were enrolled. Patients were randomized to receive Canakinumab, a therapeutic monoclonal antibody targeting interleukin-1 $\beta$, or placebo. After 48 months, the median reduction in the C-reactive protein level was greater than in the placebo group, although Canakinumab did not lead to lipid levels reduction. Importantly, the 150-mg dose of Canakinumab, managed to meet both the primary the secondary end point, resulting in reduced risk of nonfatal myocardial infarction. On the basis of these data, Canakinumab at a dose of $150 \mathrm{mg}$ every 3 months constitutes a promising therapy for recurrent cardiovascular events, independent of reducing the lipid-status. Furthermore, Ridker et al investigated whereas interleukin-6 (IL-6) inhibition is associated with cardiovascular outcomes (Table 1 , entry 5). ${ }^{22}$ During this study, 4833 patients with atherosclerosis have participated. The IL-6 levels of the patients were measured before randomization and after receiving either placebo or Canakinumab for 5 years. According to the results participants treated with Canakinumab achieved IL-6 levels below the study median level and reduction in major adverse cardiovascular events. However, in patients that IL-6 levels remained equal or above the study median level after taking the first dose of Canakinumab did not present any notable benefit concerning cardiovascular events. Therefore, there are promising results that modulation of the IL-6 signaling pathway, during therapy with Canakinumab, is related to reduction in cardiovascular event rates. Finally, Russell et al also tested the possible impact of canakinumab in patients suffering from peripheral artery disease (Table 1 , entry 6 ). ${ }^{23}$ They administered to 38 patients to a ratio of 1:1 canakinumab and placebo subcutaneously in a monthly basis. They finally demonstrated that inflammatory markers such as CPR and IL-6 fell, as well as no plaque progression, were observed in patients receiving canakinumab.

Methotrexate was also tested for possible antiatherogenetic effect on patients with cardiovascular diseases (Table 1, entry 7). ${ }^{24}$ Patients received low doses of methotrexate weekly or placebo. The primary endpoint of the study was nonfatal stroke, nonfatal myocardial infraction or sudden cardiovascular death. The use of methotrexate was not associated with fewer levels of inflammatory cytokines such as CRP, IL-1, IL-6 and did not reduce the risk for a cardiovascular event. In addition, side effects, including elevation of liver enzymes, leucopenia and anemia, occurred.

\section{Protein Kinase Inhibitors}

The mitogen-activated protein kinases (MAPKs) mediate inflammatory pathways that are associated with atherosclerosis such as the production of cytokines. The most studied mitogen-activated protein kinase is the p38 MAPK, utilizing the inhibitor Losmapimod. It has already been reported that Losmapimod attenuates the inflammatory response in the vascular wall and as a result, stabilizes plaque and decreases the rate of inflammation C-reactive protein. Thus, it demonstrates a clinical benefit.

In 2012, Elkhawad et al studied the correlation of the p38 mitogen-activated protein kinase cascade to the initiation and progression of inflammatory diseases, including atherosclerosis (Table 1 , entry 8 ). ${ }^{25}$ During this study, patients with atherosclerosis, treated with statin therapy, were randomized to receive Losmapimod or placebo. After 84 days, the vascular inflammation was detected by ${ }^{18} \mathrm{~F}$-fluorodeoxyglucose positron emission tomography or computed tomography imaging of the carotid arteries and aorta. According to the results, a significant reduction in average tissue-to-background ratio was observed in patients, who received a high dose of Losmapimod, compared to placebo. A notable reduction in inflammatory biomarkers and ${ }^{18} \mathrm{~F}$-fluorodeoxyglucose uptake in visceral fat was also detected in patients treated with Losmapimod. As a result, a high dose of Losmapimod led to the reduction of vascular inflammation in the most inflamed regions, as well as to the reduction in inflammatory biomarkers and ${ }^{18} \mathrm{~F}$-fluorodeoxyglucose uptake in visceral fat, indicating a systemic anti-inflammatory action.

In 2014, Newby et al investigated the potential of p38 MAPK inhibition concerning myocardial protective effects (Table 1 , entry 9). ${ }^{26}$ In this trial, patients with non-STsegment elevation myocardial infarction (NSTEMI) were treated with Losmapimod or placebo. According to the results, high sensitivity $\mathrm{C}$-reactive protein concentrations at $72 \mathrm{~h}$ were detected to be lower in patients treated with Losmapimod, compared to placebo group. However, the same concentrations proved to be similar after 12 weeks treatment. Moreover, mean B-type natriuretic peptide concentrations were also similar at $72 \mathrm{~h}$ of treatment, but notably lower in patients received Losmapimod at 12 weeks. Additionally, mean troponin I area under the curve values did not present any difference. On the basis of these data, p38 MAPK inhibition with oral Losmapimod can be considered as a well-tolerated and efficient treatment method in NSTEMI patients and can 
result in beneficial outcomes after acute coronary syndromes.

Losmapimod was also examined by O'Donoghue et al. They conducted a trial, in which 25.500 patients presenting non-ST-elevation or ST-elevation myocardial infarction (MI) participated. ${ }^{27}$ Patients were randomized to receive oral Losmapimod or placebo for 24 weeks. The end point of this trial is the prevention of cardiovascular death, myocardial infarction, or severe recurrent ischemia. The clinical results reported in 2016 (Table 1 , entry 10), ${ }^{28}$ according to which, patients in both groups presented similar clinical outcomes since $99.1 \%$ of the patients had complete ascertainment for the composite of cardiovascular death, MI, or severe recurrent ischemia. More specifically, the primary end point was observed by 12 weeks in $7 \%$ of the patients treated with placebo and $8 \%$ of the patients treated with Losmapimod. Treatment rates of serious adverse events were detected in $16 \%$ of the patients treated with Losmapimod and in $14 \%$ of the patients treated with placebo.

\section{Colchicine}

Colchicine is a widely available and low-cost therapeutic tool with various anti-inflammatory properties. Blocking the NLRP3 inflammasome, a cytosolic complex responsible for the production of interleukins IL-1b and IL-18, Colchicine is considered as a suitable treatment method for the prevention of atherosclerosis.

Firstly, Raju et al examined the administration of colchicine $1 \mathrm{mg}$ or placebo in 80 patients with acute coronary syndrome or ischemic stroke (Table 1, entry 11). ${ }^{29}$ They compared the effects of the above drugs on CRP and platelet function. They showed no difference in both clinical and laboratory findings. However, an increase in the incidence of diarrheas was observed. Another study of Kajikawa et al also showed no promising results concerning the use of colchicines on endothelial function and atherosclerosis (Table 1, entry 12). ${ }^{30}$ They conducted a trial of 28 patients with acute coronary syndrome, who received placebo or colchicines of $0.5 \mathrm{mg}$ orally for 7 days. Although a reduction of CRP was shown in the colchicines group, no significant difference in the flow mediated vasodilation was noticed $(\mathrm{p}=0.384)$.

On the contrary, other clinical trials showed encouraging results of the use of colchicines in cardiovascular diseases. In 2013, Nidorf et al investigated whereas Colchicine at a dose of $0.5 \mathrm{mg} /$ day is able to reduce the risk of cardiovascular episodes in patients with coronary disease (Table 1, entry 13). ${ }^{31}$ In this clinical trial, 532 patients with stable coronary disease were enrolled. Patients, who were already receiving Aspirin, Clopidogrel and statins were randomized to be treated with Colchicine $0.5 \mathrm{mg}$ /day or no Colchicine for 3 years. In the $5.3 \%$ of the patients treated with Colchicine and in $16 \%$ of the patients who did not receive Colchicine, a composite incidence of acute coronary syndrome, outof-hospital cardiac arrest, or non-cardioembolic ischemic stroke was observed. As a result, Colchicine in a dose of $0.5 \mathrm{mg} /$ day in addition to statins proved to be a promising therapeutic option for the prevention of cardiovascular events in patients with coronary disease.

Furthermore, Martínez et al conducted a trial in order to estimate the cardiac production of inflammatory cytokines in patients with acute coronary syndromes and to investigate the ability of Colchicine to prevent their production (Table 1, entry 14). ${ }^{32}$ During this trial, 33 patients suffering from stable coronary artery disease and 10 control subjects participated. Coronary artery disease patients were randomized to colchicine or no colchicine, 6 to 24 hours before cardiac catheterization. IL-1b, IL-18, and IL6 were detected in blood samples, using ELISA. In patients suffering from coronary artery disease, coronary sinus levels of IL-1b, IL-18, and IL-6 were significantly higher than arterial and venous. Trans coronary levels of IL-1b, IL-18, and IL-6 were highest in coronary artery disease patients and lowest in controls. According to the results, treatment with Colchicine led to significantly reduced trans coronary levels of these cytokines in coronary artery disease patients by $40 \%$ to $88 \%$ and can be considered as a potential treatment option for increased local cardiac production of inflammatory cytokines in these patients. Another study also tested the possible effect of colchicines in patients with acute coronary syndrome (Table 1, entry 15). ${ }^{33}$ Specifically, 21 patients were randomized to either placebo or colchicines orally at 2 doses, with a difference of 1 hour. Blood specimens were examined. The researchers demonstrated reduced levels of IL-1 and caspase- 1 and pro-caspase- 1 levels in patients receiving colchicine.

In 2017, Vaidya et al conducted a study in order to evaluate the impact of Colchicine on the plaque modification, as well as the optimal medical therapy in patients that had underwent an acute coronary syndrome (Table 1, entry 16). ${ }^{34}$ In this study 80 patients presented with acute coronary syndrome randomized to receive either $0.5 \mathrm{mg} /$ day Colchicine plus the optimal medical therapy or the optimal 
medical therapy alone for 1 year. Then, low attenuation plaque volume (LAPV), a marker of plaque instability was detected via coronary computed tomography angiography. Also, changes in high-sensitivity C-reactive protein (hsCRP) were also investigated. According to the results, Colchicine therapy led to a significant reduction of LAPV and hsCRP was observed, compared to controls. Concerning the total atheroma volume and low-density lipoprotein levels, the results were similar. In the case of multivariate linear regression, patients that received Colchicine presented significantly decreased levels in LAPV and hsCRP. As a result, low-dose Colchicine therapy can be beneficial and positively modify the coronary plaque. Given that Colchicine has anti-inflammatory properties, as proved by reductions in hsCRP, the clinical findings of this study suggest that Colchicine may be meaningful as an additional secondary treatment option in patients having presented acute coronary syndrome.

Tucker et al examined the possible impact of colchicines administration in patients with acute coronary syndrome (Table 1, entry 17). ${ }^{35}$ Patients received orally colchicines $1.5 \mathrm{mg}$ daily. They measured the levels of specific inflammatory molecules after cardiac catheterization. They showed that trans coronary levels of chemokine ligand 2 (CCL2), C-X3-C motif chemokine ligand 1 (CX3CL1) were less elevated in patients receiving colchicines compared to patients receiving placebo. Another study came to confirm the above results (Table 1, entry 18). ${ }^{36}$ Specifically, colchicine of $0.5 \mathrm{mg}$ was given to patients suffering from acute coronary infraction the last 30 days. The daily administration was associated with lower risk of cardiovascular events such as angina, myocardial infraction and death.

This year, a randomized double-blinded controlled trial examined the results of the administration of colchicines $0.6 \mathrm{mg}$ or placebo twice daily in 40 subjects with metabolic syndrome or obesity (Table 1, entry 19). ${ }^{37}$ The researchers showed that colchicine reduced levels of atherogenetic and inflammatory factors such as IL-6. They increased levels of molecules, which help damage repair such as growth/differentiation factor 15 and antithrombotic proteins, such as protein $\mathrm{C}$. Decrease in fasting insulin resistance was highly associated with $\operatorname{GDF} 15(\rho=-0.63$, $p=0.006)$.

\section{P-Selectin}

A sign of inflammation is the recruitment of leukocytes, which depends on the expression of endothelial- leukocytes. This process is based not only on endothelial cells but also on an adhesion molecule, known as P-Selectin. This adhesion molecule plays a crucial role in acute inflammation complicated by thrombosis.

In 2013, Tardif et al investigated whereas Inclacumab, a recombinant monoclonal antibody against P-Selectin, can be considered as a potential therapeutic agent for the reduction of myocardial damage, that may appear in the case of percutaneous coronary intervention, in patients with non-ST segment elevation myocardial infarction (Table 1, entry 20). ${ }^{38}$ During this trial, 544 patients with non-ST segment elevation myocardial infarction participated and were randomized in a 1:1:1 ratio to 3 groups: the first group was treated with Inclacumabin a dose of $5 \mathrm{mg} / \mathrm{kg}$, the second group was treated with Inclacumabin a dose of $20 \mathrm{mg} / \mathrm{kg}$ and the third group received placebo (SELECT-ACS Trial). Then the change from baseline in troponin I (TnI) and in CK-MB, after 16 and 24 hours after percutaneous coronary intervention, was detected. According to the results, Inclacumabin a dose of $5 \mathrm{mg} / \mathrm{kg}$ did not lead to any change concerning the TnI level. However, Inclacumabin a dose of $20 \mathrm{mg} / \mathrm{kg}$ resulted in a significant decrease in TnI levels, compared to placebo. Along the same lines, CK-MB levels were reduced in the group treated with Inclacumab in a dose of $20 \mathrm{mg} / \mathrm{kg}$, compared to the placebo group. As a result, Inclacumab appears to be a potential and promising treatment option for the reduction of myocardial damage after PCI in patients with NSTEMI.

In 2016, Stähli et al studied the impact of Inclacumab on myocardial damage (Table 1, entry 21 ). ${ }^{39}$ In this study, varying time periods were passed between the drug infusion and percutaneous coronary intervention. Patients have been enrolled in the SELECT-ACS trial, presented above, and randomized to be treated with Inclacumab ( 5 or $20 \mathrm{mg} / \mathrm{kg}$ ) or placebo, were divided based on the time interval between the drug infusion and PCI. The change of troponin I levels from baseline at 16 and 24 hours after PCI was detected. Patients treated with Inclacumab $20 \mathrm{mg} / \mathrm{kg}$ with a short time interval between infusion and PCI, the changes in troponin I and creatine kinase-myocardial band proved to be decreased. Troponin I and creatine kinase-myocardial band levels in patients with a long time interval between infusion and PCI were observed to be similar to the initial ones. Therefore, Inclacumab in a dose of $20 \mathrm{mg} / \mathrm{kg}$ notably decrease myocardial damage after percutaneous coronary intervention in patients with non-ST-segment elevation myocardial infarction and seems to be more beneficial in the case that the drug infusion takes place less than 3 hours before PCI. 


\section{NLRP3 Inhibitors}

NLR family, that includes pyrin domain-containing protein 3 (NLRP3) inflammasome, is a multi-protein complex, playing a crucial role in the mediation of innate inflammatory responses that are related to numerous pathogenassociated pathways, including atherosclerosis. Concerning the molecular pathways, identification of altered LDL particles by toll-like receptors (TLRs) or scavenger receptors on macrophages trigger the activation of NLRP3 complex in atherosclerosis.

This activation leads to the activation of ProIL-1 $\beta$ and caspase-1 that are also up-regulated. Then, the modified LDL particles initiate the formation of cholesterol crystal in macrophages. Since cholesterol levels are increased in the macrophage cell, and they become "foam cells". Lysosomal rupture takes place releasing cathepsins release. As a result, the formation of atherosclerotic plaques is initiated. Therefore, several studies have highlighted the therapeutic potential of NLRP3 inflammasome activation inhibitors, concerning the development and progression of atherosclerosis.

In 2015, Coll et al presented the development of MCC950, a selective agent that acts as an inhibitor of NLRP3 (Table 1, entry 22). ${ }^{40}$ In this study, mouse bone macrophages derived from the marrow, human macrophages from monocytes as well as human peripheral blood mononuclear cells were used in order the impact of MCC950 on NLRP3 inflammasome activation to be evaluated. Importantly, MCC950 has the ability to prevent the canonical and non-canonical NLRP3 activation even when it is used at nanomolar concentrations. According to the results, MCC950 specifically blocked the activation of NLRP3 inflammasome, but he proved to be unable to block the activation of the AIM2, NLRC4 or NLRP1 inflammasomes. MCC950 also led to the reduction of interleukin-1b (IL-1b) production in vivo and resulting in less severe autoimmune encephalomyelitis that was developed experimentally in a model subject with multiple sclerosis. Moreover, MCC950 was found to reduce neonatal lethality in a mouse model of CAPS. As a result, MCC950 is considered as a potential therapeutic option for NLRP3-related syndromes, such as auto-inflammatory diseases.

\section{Discussion}

Atherosclerosis, being the leading cause of several cardiovascular conditions, such as heart attacks, strokes, and peripheral vascular disease, is a quite important disorder.
Therefore, numerous different therapeutic strategies have been developed, based on the efficiency of immunotherapeutic interventions, aiming to the decrease atherosclerosis levels. ${ }^{41}$

Several already existing reviews have presented a plethora of trials, evaluating the efficiency and the safety of the demonstrated therapeutic strategies. For example, the antithrombotic treatment and antiplatelet or anticoagulant therapeutic agents have been reported to play an important role in the treatment of atherosclerosis. ${ }^{42}$ A very common drug, used in antiplatelet therapy, is aspirin which is related to a significant reduction in the risk of myocardial infarction, presenting anti-inflammatory properties. Other antiplatelet drugs that have been used for the management of atherosclerosis are thienopyridines. However, the antiatherogenic effects of antiplatelet drugs are controversial, since are not confirmed from clinical trials.

A therapeutic agent against atherosclerosis that has been widely studied is interleukin-1 beta (IL-1b). ${ }^{43,44}$ The mechanistic pathway is based on the generation of active IL-1b via the action of a molecular complex, also known as the inflammasome by local stimuli in the plaque favor. A variety of clinical findings suggest that treatment that inhibits the IL-1 action lead to improved cardiovascular outcomes. Others treatment methods that have been suggested for the management of atherosclerosis are various antioxidants which have not been proved quite beneficial in the clinical trials. ${ }^{45}$ Furthermore, the p38 mitogenactivated protein kinase (MAPK) inhibitor, P-Selectin, which is an inhibitor of a leukocyte adhesion, as well as low-dose methotrexate has been tested in several clinical trials with leading to mediocre clinical findings. ${ }^{45}$

Some studies suggest that agents blocking the absorption of dietary cholesterol lead to an increase in HDL-cholesterol and a decrease in LDL-cholesterol. As a result a reduction in triglyceride levels is achieved that has been proved to be beneficial in the treatment of atherosclerosis. ${ }^{46}$ Statins being therapeutic agents that are associated with low-density lipoprotein (LDL) can reduce the risk of atherosclerotic events. Statins have been proved to reduce the levels of highsensitivity $\mathrm{C}$-reactive protein and adhesion molecules that are increased in inflammation conditions and atherosclerosis. ${ }^{47,48}$ Apart from statins, proprotein convertase subtilisin/kexin type 9 (PCSK9) inhibitors have been shown to reduce LDL-C levels when administered as a treatment option alone or in combination with statins. As a result, PCSK9 inhibitors can actually reduce the incidence 
of cardiovascular conditions, including atherosclerosis. ${ }^{49}$ Incretin-based therapies have also been developed and are reported to be effective and well tolerated, leading to lowering the risk of cardiovascular events. ${ }^{50}$

Herein, the therapeutic potential of several cytokines, protein kinase inhibitors, Colchicine, p-Selectin and an inhibitor of NLRP3 is discussed, based on studies that have been conducted from the time period 2010-2020. According to the results, cytokines participating in the inflammatory pathway of atherosclerosis, led to the most promising results. More specifically, agents that block Interleukins, which are pro-inflammatory cytokines, such as Canakinumab have been used as therapeutic tools for the reduction of atherosclerosis, presenting safety, tolerability and efficacy. Additionally, Glycoprotein 130 (sgp130) and IgG1-Fc (sgp130Fc), that are interleukin-6 (IL-6) inhibitors, have been proved to reduce advanced atherosclerosis in hypercholesterolemic mice, suggesting that IL- 6 transsignaling may be an option for the managing of atherogenesis in humans. Moreover, IL-1 $\alpha$ proved to be the predominant inflammatory cytokine related to atherosclerosis by IL-1R signaling. Therefore, Xilonix, a true human monoclonal antibody targeting IL-1a, can be considered as a potential treatment agent safe and effective therapeutic method for major adverse cardiovascular events, including atherosclerosis.

The mitogen-activated protein kinases (MAPKs) mediate inflammatory pathways are linked to atherosclerosis. Losmapimod, the most widely known mitogen-activated protein kinase inhibitor, is considered to be a suitable treatment option for the reduction of vascular inflammation when it is used in high doses. Losmapimod proved to be a well tolerated and efficient therapeutic agent for patients with acute coronary syndromes.

Apart from cytokines and protein kinases inhibitors, Colchicine is a therapeutic tool with a variety of antiinflammatory properties. According to the presented trials, Colchicine in a low dose therapy can be a promising therapeutic option for the prevention of cardiovascular events in patients with coronary disease. More beneficial clinical results are achieved when Colchicine is used in combination with statins.

Inflammation occurs with the recruitment of leukocytes, which is associated with an adhesion molecule, P-Selectin. Inclacumab is a recombinant monoclonal antibody against P-Selectin and has been suggested as a potential and meaningful treatment option for the decrease of myocardial damage after PCI in patients with
NSTEMI, reducing troponin I (TnI) and in CK-MB levels. However, Inclacumab seems to be more beneficial when the drug infusion is done less than 3 hours before PCI.

Inhibitors of NLRP3, a pyrin domain-containing protein 3 inflammasome related to various pathogenassociated disorders, including atherosclerosis. A selective inhibitor of NLRP3 is MCC950, which results in the reduction of interleukin-1b production and reduced the severity of autoimmune encephalomyelitis developed in a model subject with multiple sclerosis. MCC950 also reduces neonatal lethality in a mouse model of CAPS.

Finally, molecular imaging of atherosclerotic lesions constitutes a crucial experimental tool nowadays, providing a direct insight to the molecular pathway of atherosclerosis and its complications. ${ }^{51}$ Molecular imaging should be in wide use during the clinical trials in order highlights on the cardiovascular process to be achieved. Thus, this will lead to the development and evaluation of novel, alternative and promising therapeutics.

\section{Conclusion}

In conclusion, the therapeutic potential of cytokines, protein kinase inhibitors, Colchicine, p-Selectin and NLRP3 inhibitors is discussed. These agents gain ground in prevention of atherosclerosis, since beneficial clinical results are achieved. However, reliable biomarkers for efficient interventions need to be defined, in order for successful monitoring of such cardiovascular disorders like atherosclerosis to be achieved. Moreover, a deeper understanding of the mechanism of this disorder may lead to the development of suitable intervention mediates for this immune response. More clinical trials should also be conducted, for avoiding any biased conclusion concerning the evaluation of the clinical outcomes.

\section{Disclosure}

The authors report no conflicts of interest in this work.

\section{References}

1. Hansson GK, Hermansson A. The immune system in atherosclerosis. Nat Immunol. 2011;12(3):204-212. doi:10.1038/ni.2001

2. Murray CJ, Lopez AD. Alternative projections of mortality and disability by cause 1990-2020: global burden of disease study. Lancet. 1997;349(9064):1498-1504. doi:10.1016/S0140-6736(96)07492-2

3. Hopkins PN, Williams RR. A survey of 246 suggested coronary risk factors. Atherosclerosis. 1981;40:1-52.

4. Kannel WB, Wilson PW. An update on coronary risk factors. Med Clin North Am. 1995;79(5):951-971. doi:10.1016/S0025-7125(16)30016-5

5. Glass CK, Witztum JL. Atherosclerosis. The road ahead. Cell. 2001;104(4):503-516. doi:10.1016/S0092-8674(01)00238-0 
6. Hansson GK, Libby P. The immune response in atherosclerosis: a double-edged sword. Nat Rev Immunol. 2006;6(7):508-519. doi:10. $1038 /$ nri1882

7. Dinarello CA. Interleukin-1 in the pathogenesis and treatment of inflammatory diseases. Blood. 2011;117(14):3720-3732. doi:10.11 82/blood-2010-07-273417

8. Feldmann M. Many cytokines are very useful therapeutic targets in disease. J Clin Invest. 2008;118(11):3533-3536. doi:10.1172/JCI37346

9. Ait-Oufella H, Taleb S, Mallat Z, et al. Recent advances on the role of cytokines in atherosclerosis. Arterioscler Thromb Vasc Biol. 2011;31(5):969-979. doi:10.1161/ATVBAHA.110.207415

10. Chatenoud L, Thervet E, Primo J, et al. Anti-CD3 antibody induces long-term remission of overt autoimmunity in nonobese diabetic mice. Proc Natl Acad Sci U S A. 1994;91(1):123-127. doi:10.1073/ pnas.91.1.123

11. Steffens S, Burger F, Pelli G, et al. Short-term treatment with anti-CD3 antibody reduces the development and progression of atherosclerosis in mice. Circulation. 2006;114(18):1977-1984. doi:10. 1161/CIRCULATIONAHA.106.627430

12. Mach F, Schönbeck U, Sukhova GK, et al. Reduction of atherosclerosis in mice by inhibition of CD40 signalling. Nature. 1998;394 (6689):200-203. doi:10.1038/28204

13. Schönbeck U, Sukhova GK, Shimizu K, et al. Inhibition of CD40 signaling limits evolution of established atherosclerosis in mice. Proc Natl Acad Sci US A. 2000;97(13):7458-7463. doi:10.1073/pnas. 97.13.7458

14. Lutgens E, Cleutjens KB, Heeneman S, et al. Both early and delayed anti-CD40L antibody treatment induces a stable plaque phenotype. Proc Natl Acad Sci US A. 2000;97(13):7464-7469. doi:10.1073/ pnas.97.13.7464

15. Wick G, Knoflach M, Xu Q. Autoimmune and inflammatory mechanisms in atherosclerosis. Annu Rev Immunol. 2004;22(1):361-403. doi:10.1146/annurev.immunol.22.012703.104644

16. Hansson GK, Robertson AK, Söderberg-Nauclér C. Inflammation and atherosclerosis. Annu Rev Pathol. 2006;1(1):297-329. doi:10. 1146/annurev.pathol.1.110304.100100

17. Ridker PM, Thuren T, Zalewski A, et al. Interleukin-1 $\beta$ inhibition and the prevention of recurrent cardiovascular events: rationale and design of the canakinumab anti-inflammatory thrombosis outcomes study (CANTOS). Am Heart J. 2011;162(4):597-605. doi:10.1016/j. ahj.2011.06.012

18. Schuett H, Oestreich R, Waetzig GH, et al. Transsignaling of interleukin-6 crucially contributes to atherosclerosis in mice. Arterioscler Thromb Vasc Biol. 2012;32(2):281-290. doi:10.1161/ ATVBAHA.111.229435

19. Freigang S, Ampenberger F, Weiss A, et al. Fatty acid-induced mitochondrial uncoupling elicits inflammasome-independent IL- $1 \alpha$ and sterile vascular inflammation in atherosclerosis. Nat Immunol. 2013;14(10):1045-1053. doi:10.1038/ni.2704

20. El Sayed H, Kerensky R, Stecher M, et al. A randomized Phase II study of Xilonix, a targeted therapy against interleukin $1 \alpha$, for the prevention of superficial femoral artery restenosis after percutaneous revascularization. J Vasc Surg. 2016;63(1):133-141. doi:10.1016/j. jvs.2015.08.069

21. Ridker PM, Everett BM, Thuren T, et al. Antiinflammatory therapy with canakinumab for atherosclerotic disease. $N$ Engl J Med. 2017;377(12):1119-1131. doi:10.1056/NEJMoa1707914

22. Ridker PM, Libby P, MacFadyen JG, et al. Modulation of the interleukin-6 signalling pathway and incidence rates of atherosclerotic events and all-cause mortality: analyses from the canakinumab anti-inflammatory thrombosis outcomes study (CANTOS). Eur Heart J. 2018;39(38):3499-3507. doi:10.1093/eurheartj/ehy310

23. Russell KS, Yates DP, Kramer CM, et al. A randomized, placebo-controlled trial of canakinumab in patients with peripheral artery disease. Vasc Med. 2019;24(5):414-421. doi:10.1177/1358863 X19859072
24. Ridker PM, Everett BM, Pradhan A. Low-dose methotrexate for the prevention of atherosclerotic events. $N$ Engl J Med. 2019;380 (8):752-762. doi:10.1056/NEJMoa1809798

25. Elkhawad M, Rudd JH, Sarov-Blat L, et al. Effects of p38 mitogen-activated protein kinase inhibition on vascular and systemic inflammation in patients with atherosclerosis. JACC Cardiovasc Imaging. 2012;5(9):911-922. doi:10.1016/j.jcmg.2012.02.016

26. Newby LK, Marber MS, Melloni C, et al. Losmapimod, a novel p38 mitogen-activated protein kinase inhibitor, in non-ST-segment elevation myocardial infarction: a randomised Phase 2 trial. Lancet. 2014;384(9949):1187-1195. doi:10.1016/S0140-6736(14)60417-7

27. O'Donoghue ML, Glaser R, Aylward PE, et al. Rationale and design of the LosmApimod to inhibit p38 MAP kinase as a TherapeUtic target and moDify outcomes after an acute coronary syndrome trial. Am Heart J. 2015;169(5):622-630. doi:10.1016/j. ahj.2015.02.012

28. O’Donoghue ML, Glaser R, Cavender MA, et al. Effect of losmapimod on cardiovascular outcomes in patients hospitalized with acute myocardial infarction: a randomized clinical trial. JAMA. 2016;315 (15):1591-1599. doi:10.1001/jama.2016.3609

29. Raju NC, Yi Q, Nidorf M, et al. Effect of colchicine compared with placebo on high sensitivity $\mathrm{C}$-reactive protein in patients with acute coronary syndrome or acute stroke: a pilot randomized controlled trial. J Thromb Thrombolysis. 2012;33(1):88-94. doi:10.1007/ s11239-011-0637-y

30. Kajikawa M, Higashi Y, Tomiyama H, et al. Effect of short-term colchicine treatment on endothelial function in patients with coronary artery disease. Int $J$ Cardiol. 2019;281:35-39. doi:10.1016/j. ijcard.2019.01.054

31. Nidorf SM, Eikelboom JW, Budgeon CA, et al. Low-dose colchicine for secondary prevention of cardiovascular disease. J Am Coll Cardiol. 2013;61(4):404-410. doi:10.1016/j.jacc.2012.10.027

32. Martínez GJ, Robertson S, Barraclough J, et al. Colchicine acutely suppresses local cardiac production of inflammatory cytokines in patients with an acute coronary syndrome. $J$ Am Heart Assoc. 2015;4(8):e002128. doi:10.1161/JAHA.115.002128

33. Robertson S, Martínez GJ, Payet CA, et al. Colchicine therapy in acute coronary syndrome patients acts on caspase-1 to suppress NLRP3 inflammasome monocyte activation. Clin Sci (Lond). 2016;130(14):1237-1246. doi:10.1042/CS20160090

34. Vaidya K, Arnott C, Martínez GJ, et al. Colchicine therapy and plaque stabilization in patients with acute coronary syndrome: a CT coronary angiography study. JACC Cardiovasc Imaging. 2018;11 (2):305-316. doi:10.1016/j.jcmg.2017.08.013

35. Tucker B, Kurup R, Barraclough J, et al. Colchicine as a novel therapy for suppressing chemokine production in patients with an acute coronary syndrome: a pilot study. Clin Ther. 2019;41 (10):2172-2181. doi:10.1016/j.clinthera.2019.07.015

36. Tardif JC, Kouz S, Waters DD, et al. Efficacy and safety of low-dose colchicine after myocardial infarction. $N$ Engl J Med. 2019;381 (26):2497-2505. doi:10.1056/NEJMoa1912388

37. Demidowich AP, Levine JA, Apps R, et al. Colchicine's effects on metabolic and inflammatory molecules in adults with obesity and metabolic syndrome: results from a pilot randomized controlled trial. Int $J$ Obes (Lond). 2020;44(8):1793-1799. doi:10.1038/ s41366-020-0598-3

38. Tardif JC, Tanguay JF, Wright SR, et al. Effects of the P-selectin antagonist inclacumab on myocardial damage after percutaneous coronary intervention for non-ST-segment elevation myocardial infarction: results of the SELECT-ACS trial. J Am Coll Cardiol. 2013;61(20):2048-2055. doi:10.1016/j.jacc.2013.03.003

39. Stähli BE, Gebhard C, Duchatelle V, et al. Effects of the p-selectin antagonist inclacumab on myocardial damage after percutaneous coronary intervention according to timing of infusion: insights from the SELECT-ACS trial. J Am Heart Assoc. 2016;5(11):e004255. doi:10.1161/JAHA.116.004255 
40. Coll RC, Robertson AA, Chae JJ, et al. A small-molecule inhibitor of the NLRP3 inflammasome for the treatment of inflammatory diseases. Nat Med. 2015;21(3):248-255. doi:10.1038/nm.3806

41. Amir S, Binder CJ. Experimental immunotherapeutic approaches for atherosclerosis. Clin Immunol. 2010;134(1):66-79. doi:10.1016/j. clim.2009.07.009

42. Jawień J. Atherosclerosis in 2012: what is new? Pol Arch Med Wewn. 2012;122(4):170-173. doi:10.20452/pamw.1192

43. Sheedy FJ, Moore KJ. IL-1 signaling in atherosclerosis: sibling rivalry. Nat Immunol. 2013;14(10):1030-1032. doi:10.1038/ni.2711

44. Libby P. Interleukin-1 beta as a target for atherosclerosis therapy: biological basis of CANTOS and beyond. $\mathrm{J} \mathrm{Am}$ Coll Cardiol. 2017;70(18):2278-2289. doi:10.1016/j.jacc.2017.09.028

45. Libby P, Everett BM. Novel antiatherosclerotic therapies. Arterioscler Thromb Vasc Biol. 2019;39(4):538-545. doi:10.1161/ ATVBAHA.118.310958

46. Saini HK, Xu YJ, Arneja AS, et al. Pharmacological basis of different targets for the treatment of atherosclerosis. J Cell Mol Med. 2005;9 (4):818-839. doi:10.1111/j.1582-4934.2005.tb00382.x
47. Diamantis E, Kyriakos G, Quiles-Sanchez LV, et al. The anti-inflammatory effects of statins on coronary artery disease: an updated review of the literature. Curr Cardiol Rev. 2017;13 (3):209-216. doi:10.2174/1573403X13666170426104611

48. Diamantis E, Troupis T, Mazarakis A, et al. Primary and secondary prevention of acute coronary syndromes: the role of the statins. Recent Adv Cardiovasc Drug Discov. 2014;9(2):97-105. doi:10. 2174/1574890110666150708112349

49. Farmaki P, Damaskos C, Garmpis N, et al. PCSK9 inhibitors and cardiovascular disease: impact on cardiovascular outcomes. Curr Drug Discov Technol. 2020;17(2):138-146. doi:10.2174/15701638 16666181211112358

50. Kyriakos G, Quiles-Sanchez LV, Garmpi A, et al. Cardiovascular and renal outcomes of incretin-based therapies: a review of recent clinical trials. Curr Cardiol Rev. 2019. doi:10.2174/1573403X1566619 0603111056

51. Quillard T, Libby P. Molecular imaging of atherosclerosis for improving diagnostic and therapeutic development. Circ Res. 2012;111(2):231-244. doi:10.1161/CIRCRESAHA.112.268144

\section{Publish your work in this journal}

The Journal of Experimental Pharmacology is an international, peerreviewed, open access journal publishing original research, reports, reviews and commentaries on all areas of laboratory and experimental pharmacology. The manuscript management system is completely online and includes a very quick and fair peer-review system. Visit http://www.dovepress.com/testimonials.php to read real quotes from published authors.

Submit your manuscript here: https://www.dovepress.com/journal-of-experimental-pharmacology-journal 